\section{Cable tension identification via nonlinear static inverse problem}

Structural Health Monitoring $|-2|$

(C) The Author(s) 2020

Article reuse guidelines: sagepub.com/journals-permissions DOI: I0.I I 77//47592 I 720940645 journals.sagepub.com/home/shm (SAGE

\author{
Arnaud Pacitti' ${ }^{1}$, Michaël Peigney $^{2}$, Frédéric Bourquin ${ }^{3}$ \\ and Walter Lacarbonara ${ }^{4}$
}

\begin{abstract}
A new identification technique is proposed to evaluate the tension of a cable using a static inverse approach that couples a universal cable model with displacement sensors, strain gauges and added masses that should preserve operational affordability. An inverse problem is formulated as the minimization of a data misfit functional based on the differences in terms of vertical displacements and axial strains between two equilibrium configurations of the cable, namely, one loaded and the other free. The inverse problem formulation echoes the parametric study of a non-conventional functional suggesting a way to identify the cable parameters, namely, its length, its axial stiffness, and its mass per unit length. The computational resolution of the inverse problem is implemented as a two-step identification procedure. First, the axial stiffness and mass per unit length are kept constant and the length of the cable is approximately found via a simple line search algorithm using finite differences to estimate the functional derivatives. Second, the other physical parameters are assessed using an adjoint method for which the direct problem, the adjoint problem, and the parameter sensitivities are defined as derivatives of a Lagrangian functional with respect to dual variables, primal variables, and parameters, respectively. Due to the ill-conditioning of the problem, the proposed method does not enable an exact parameter identification but yields a good tension assessment. An experimental test campaign conducted on a multilayered $2 \mathrm{l}-\mathrm{m}$ long stranded cable subject to several tension levels confirms the relevance of the proposed inverse method. A field test campaign of the method on three 120-m long cables of Bonny-sur-Loire (France) suspension bridge is also presented. It proves the reliability and affordability of the overall tension identification process.
\end{abstract}

\title{
Keywords
}

Cable, mixed formulation, inverse problem, tension identification

\section{Introduction}

Because of its leading role in structural health monitoring, tension identification of bridge cables has been widely studied over the years. At the cable level, it enables one to estimate its remaining load-bearing capacity. At the bridge scale, tension assessment yields quantitative information on the structural tuning and possible malfunctioning. When no permanent monitoring system is installed on a bridge, three main methods exist for tension assessment: the use of jacks, the use of tensiometers, and dynamical testing. Using jacks on a job site is costly and more likely to happen during a repair or heavy maintenance operation (suspender or anchorage replacement, suspension tuning operation, etc.) for which an accurate tension assessment is usually not necessary.

The tensiometer can be of great help for tension identification of small cables and ropes but its response depends on the cable type, urging for laboratory calibration. It is therefore an effective tool for tension assessment in post-tensioned strands but its scope does not embrace the large diversity of cable types encountered in civil engineering.

\footnotetext{
'Département Laboratoire de Bordeaux, Cerema Sud-Ouest, Bordeaux, France

${ }^{2}$ Laboratoire Navier (UMR 8205), École des Ponts ParisTech, CNRS, IFSTTAR, Université Gustave Eiffel, Marne-la-Vallée, France ${ }^{3}$ COSYS, IFSTTAR, Université Gustave Eiffel, Marne-la-Vallée, France ${ }^{4}$ Department of Structural and Geotechnical Engineering, Sapienza University of Rome, Rome, Italy
}

Corresponding author:

Arnaud Pacitti, Mega Project Business Group, DYWIDAG-Systems International, Leipzig 04179, Germany.

Email: arnaud.pacitti@dywidag-systems.com 
Last but certainly not least, because of its application feasibility and low cost, dynamical testing for tension assessment has been extensively treated in the literature and used on sufficiently long cables. Most dynamical testing methods are based on an explicit or an implicit relationship between the tension and the natural frequencies of the cable. Despite the big differences between all the established methods in the way this relationship is found and used, they all rely on a combination of three parts: a cable model, several sensors, and data post-processing to obtain the natural frequencies of the cable. Along these lines, the most famous method uses the explicit relationship given by the string theory with accelerometers whose output is post-processed via a fast Fourier transform (FFT). In France, this method is known as the LPC 35. ${ }^{\circ}$ Zui et $\mathrm{al}^{2}$ presented a method based on a tensed clamped beam model with additional terms to take into account small sag effects of long stay cables. Because anchorage conditions impact on the dynamical behavior of short cables, three different kinds of anchorages for the tensed beam theory (clamped-clamped, hingedclamped, and hinged-hinged) were investigated by Bouton and Crémona, ${ }^{3}$ increasing the analytical developments burden but keeping the formulas easy to use. Gentile $^{4}$ used the string theory with a microwave interferometer allowing to measure the dynamic responses of several stay cables at once. Various data-processing options were investigated in order to improve the accuracy of the spectral identification, ${ }^{5}$ and richer alternatives to the conventional FFT were proposed, ${ }^{6}$ such as wavelet transforms or MUltiple SIgnal Classification (MUSIC) algorithm. A synthetic overview of dynamical testing for tension assessment ${ }^{7}$ confirms the reliability of the methods for cables longer than $19 \mathrm{~m}$.

For shorter cables, physical uncertainties due to unknown boundary conditions or physical parameters of the cable such as its length or flexural stiffness have set the tension assessment issue in terms of identification and inverse problems using tensed (and straight) beam modeling. Li et al. ${ }^{8}$ used modal shapes and length identification of a tensed beam model to identify the tension of a cable with uncertain boundary conditions. Several authors ${ }^{9-12}$ proposed an identification of stiffnesses at the boundary using the minimization of a data misfit functional taking advantage of the natural frequencies.

To the best of the authors' knowledge, very few works consider the mass per unit length as an unknown of the problem. In practice, its value is seldom known with accuracy. Its computation would request the availability of information on coating, corrosion protection system, paint, and internal structure of the cable. Depending on the case, the resulting uncertainty on the mass per unit length of cable is likely to be around
$5 \%-10 \%$. In the case of dynamical testing using the string theory, this uncertainty directly propagates to the tension identification and deteriorates the precision thereof.

In the presented work, an inverse problem is formulated as the minimization of a data misfit functional involving an observation made of the differences in terms of vertical displacements and axial strains between two equilibrium configurations of the cable: one loaded and the other free. It enables one to find the tension in a cable without knowing precisely a priori its physical parameters, namely, its (unstretched) length $L$, its mass per unit length, and its axial stiffness.

Section "Preliminaries: inverse problem and cable parameters" gives an overview on the proposed method, mixing general inverse problem theory and practical considerations on cable parameters. In section "Cable model," we introduce the data misfit designed for the static tension identification method and we present an original mixed formulation for nonlinear cables. A numerical parametric study of the data misfit is conducted and suggests a two-step tension identification procedure described in section "Data misfit." The idea of a two-step procedure to reduce the ill-conditioning of cable problems has already been used and can be found in the literature; see, for instance, Lepidi et al. ${ }^{13}$ for dynamic damage identification and Joaquim et al. ${ }^{14}$ for dynamic tension assessment. These studies do not consider the mass per unit length of cable as an unknown despite its importance in the cable dynamics. It is worth noticing that contrary to most frequencybased studies, the characterization of the static configuration is part of the inverse problem presented in Lepidi et al. ${ }^{13}$ In section "Cable model," the inverse problem is presented and its successful experimental validation is illustrated in section "Inverse problem." The methodology can be applied to a large variety of cables and its performance is investigated in section "Performance assessment of the inverse methodology." A version considering flexural stiffness has been investigated by Pacitti. ${ }^{15}$ In section "Experimental validation," an experimental validation made at Cerema's test facilities on a $21-\mathrm{m}$ long cable tensed at six different levels of tension is presented. The method proved to be operationally effective when applied to three cables of the Bonny-sur-Loire (Loiret, France) suspension bridge. The results of this successful in situ test campaign are reported in section "Application to three top cables of a suspension bridge."

All simulations were conducted in a Python environment on the computing platform FEniCS. ${ }^{16}$ Space discretization is done with $P 1$ Lagrangian finite elements for the cable positions and $P 0$ discontinuous elements for its tension and strain. Derivatives are projected at quadrature points and validation of the routines was 
performed by comparison with the closed-form solution of a strongly nonlinear three-dimensional (3D) catenary problem. ${ }^{15}$

\section{Preliminaries: inverse problem and cable parameters}

In this section, general matters on inverse problems are briefly recalled and used to give an overall picture of the proposed method. The tension assessment problem is an optimization problem that involves a state equation, a set of cable parameters, and experimental data. Let the three Hilbert spaces $\mathbb{P}, \mathbb{U}$, and $\mathbb{D}$ be the parameter space, the state space, and the data space, respectively. The state equation gives the implicit relation between the parameters and the state

$$
F(q, u)=0, \quad q \in \mathbb{P}, \quad u \in \mathbb{U}
$$

Section "Cable model" presents the elastic cable model used to express equation (1). An elastic cable is fully described by four parameters among five possible, namely, its length $L$, its axial stiffness $E A$, its mass per unit length $m$, its tension at one anchorage, and the relative positions of its anchorages. Because these latter can be easily assessed with a good accuracy in practice (e.g. with a laser sensor, or when the construction drawings are available), they are taken as a datum of the problem. The four other parameters are not precisely known. The observation equation gives the relation between the observed data and the state

$$
d=H u, \quad u \in \mathbb{U}
$$

where $H$ is the observation operator. An inverse problem aims to find the parameters $q$ such that $d=H(S(q))$, where $S(q)$ is the solution of $F(q, S(q))=0$. Let $\phi$ be the implicit function such that $\phi(q)=H(S(q))$. In practice, the problem can be rewritten as

$$
\begin{aligned}
& \text { Find } q \in \mathbb{P} \text { such that } \\
& \mathfrak{J}(q)=\min \mathfrak{J}(p)=\min \frac{1}{2}\|\phi(p)-d\|_{D}^{2} \\
& \text { for all } p \in \mathbb{P}_{q} \subset \mathbb{P}
\end{aligned}
$$

Problem (3) is well-posed according to Hadamard ${ }^{17}$ iff (1) a solution exists, (2) the solution is unique, and (3) the solution's behavior changes continuously with the initial conditions. For the tension assessment problem, requirements (1) and (3) are a priori fulfilled. On the contrary, requirement (2) is not met in most cases. Two cables laying between the same points can have indistinguishable tensions in practice, but quite different parameters $(L, m, E A)$. The first sources of practical ill-conditioning are the low sensitivities of parameters $m$ and $E A$ for taut and sagged cables, respectively. One can easily imagine that adding $+10 \%$ mass per unit length (respectively axial rigidity) to a taut cable (respectively to a sagged cable) will only induce negligible changes in its tension. The second sources of illconditioning are relative to parameters $(L, E A)$ for taut cables and $(L, m)$ for sagged cables. Two cables slightly differing in parameters $(L, E A)$ (respectively $(L, m))$ can have indistinguishable tensions if the shortest is stiffer (respectively heavier) than the longest. To cover all types of cables, the inverse problem has to circumvent requirement (2) and needs to consider the three parameters $(L, m, E A)$. One consequence of the violation of (2) is that a standard inverse problem using one state equation like equation (3) does not lead to a correct tension assessment, as illustrated in section "Setting the stage: why a standard residual does not work?" An original data misfit $\mathfrak{J}^{a, b}$ involving two state equations is introduced to overcome requirement (2). In section "Numerical exploration of the novel data misfit functional $\mathfrak{J}^{\mathfrak{a}, \mathfrak{b}}, "$ a numerical exploration of the proposed data misfit $\mathfrak{J}^{a, b}$ enlightens the problem sensitivity to the parameters $(L, m, E A)$ for three different cable configurations: one taut, one sagged, and one intermediate. Among the cable parameters, $L$ plays a preponderant role. Thus, and as described in section "Identification of the natural length of the cable," its identification is treated first, letting $\mathfrak{J}^{a, b}=\mathfrak{J}^{a, b}(L)$ and freezing the other parameters $p=(m, E A)$ to their initial guess values. Once a length $L$ has been found, the adjoint method described in section "Identification of the tension" is used to find a set of parameters $(m, E A)$ that returns the correct tension. In the following, the application of the method to problem (3) is summarized. If $u$ and $p$ are the independent variables, a Lagrangian functional of the minimization problem is built

$$
\mathfrak{L}(u, p, l)=\frac{1}{2}\|\phi(p)-d\|_{\mathbb{D}}^{2}-<l, F(p, u)>, \forall l \in \mathbb{A}
$$

where $l$ is the Lagrangian multiplier of the constraint $F(p, u)=0,<\cdot, \cdot>$ denotes the scalar product used, and $\mathbb{A}$ is the Hilbert space. The partial derivative of $\mathfrak{L}(u, p, l)$ with respect to $l$ gives the variational formulation of the state equations

$$
<\delta l, F(p, u)>=0, \forall \delta l \in \mathbb{A}
$$

The partial derivative of $\mathfrak{L}(u, p, l)$ with respect to $u$ gives the variational formulation of the adjoint equations

$$
<H \delta u, H u-d>=<l, \partial_{u} F(p, u) \delta u>, \forall \delta u \in \mathbb{U}
$$


If $u(p)$ is the solution of the direct variational problem (5) and $l$ is the solution of the adjoint problem (6), the differential of $\mathfrak{J}(p)$ reads

$$
<l, \partial_{p} F(p, u(p)) \delta p>=\mathfrak{J}_{p}(p) \delta p
$$

The subscript $p$ denotes the differentiation with respect to the parameters $p$. In finite-dimensional space, $\mathfrak{J}_{p}(p) \delta p=\nabla \mathfrak{J}(p)^{t} \delta p$. Once the gradient of $\mathfrak{J}$ is found, the sensitivities of the problem are known and the parameters can be updated. An introduction to inverse problem can be found in Bui ${ }^{18}$ or in Kern. ${ }^{19}$

\section{Cable model}

The tension is expressed in terms of its associated safety factor

$$
\gamma=\frac{A f_{y}}{\breve{N}_{\max }}
$$

where $f_{y}$ is the yield stress of the cable, $A$ its crosssectional area, and $\breve{N}_{\max }$ is the maximum value of the tension in the cable.

In this section, we briefly present the nonlinear mixed formulation modeling of cable used for tension assessment (for more details, see Pacitti ${ }^{15}$ ).

\section{Formulation of the static problem}

A cable in the Newtonian basis $\left\{\boldsymbol{e}_{1}, \boldsymbol{e}_{2}, \boldsymbol{e}_{3}\right\}$ with origin $O$ can be described in two configurations: the stress-free configuration $\mathbf{B}^{0}$ and the current configuration $\mathbf{B}$ which could be static or not. In configuration $\mathbf{B}^{0}$, the cable is described by a curve $\mathbf{C}^{0}$ parameterized by its arc length $s$. The configuration $\mathbf{B}^{0}$ can be fully described as $\mathbf{B}^{0}:=\left\{\boldsymbol{r}^{0}, s \in[0, L]\right\}$, where $L$ is the length of the cable $\mathbf{C}^{0}$ and $\boldsymbol{r}^{0}$ is the position vector of a material point $M$ of $\mathbf{C}^{0}$ at $s$. Similarly, quantities $\mathbf{C}, \breve{s}, \breve{r}$, and $L$ are defined for the current configuration $\mathbf{B}$. An illustration of configurations $\mathbf{B}^{0}$ and $\mathbf{B}$ are shown in Figure 1.

Assuming clamped (Dirichlet) boundary conditions and a linearly elastic behavior for the cable, the classical form of the static problem is cast as

$$
\left\{\begin{array}{l}
\breve{\boldsymbol{n}}_{S}+\breve{\boldsymbol{f}}=o \\
\breve{\boldsymbol{n}}=E A \breve{\Delta} \breve{\boldsymbol{r}}_{s} \\
\breve{\Delta}=\frac{1}{2}\left(\breve{\boldsymbol{r}}_{s} \cdot \breve{\boldsymbol{r}}_{s}-1\right) \\
\breve{\boldsymbol{r}}(0)=\boldsymbol{o} \text { and } \breve{\boldsymbol{r}}(L)=L_{1} \boldsymbol{e}_{1}+L_{2} \boldsymbol{e}_{2}+L_{3} \boldsymbol{e}_{3}
\end{array}\right.
$$

where $\boldsymbol{n}$ is the tension (generalized stress resultant vector) in the cable, $\boldsymbol{f}$ is the external force per unit reference length exerted on the cable, $E A$ is the axial stiffness, and $\breve{\Delta}=1 / 2\left(\breve{\boldsymbol{r}}_{s} \cdot \breve{\boldsymbol{r}}_{s}-1\right)$ is the Green strain

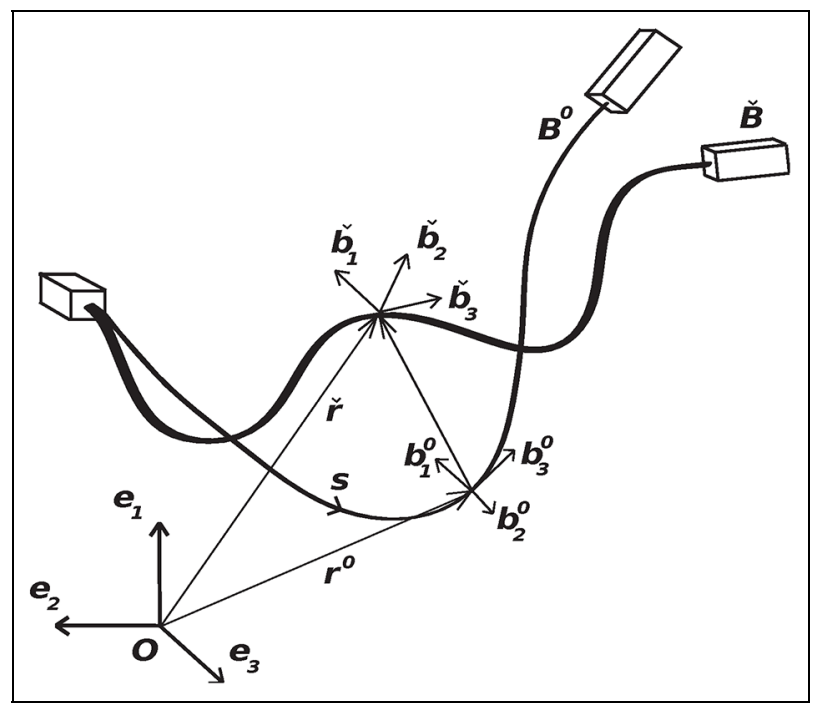

Figure I. Stress-free configuration $\mathbf{B}^{0}$ and current configuration $\mathbf{B}$.

measure. The subscript ${ }_{s}$ denotes the differentiation with respect to the arc length $s$. The parameters $\left(L_{1}, L_{2}, L_{3}\right)$ are prescribed to set the cable ends. ${ }^{20,21}$

Considering a space $H^{1}$ of sufficiently smooth displacement fields over $[0, L]$, we define the two spaces $\mathbb{V}=\left\{\breve{\boldsymbol{r}} \in H^{1} \mid \breve{\boldsymbol{r}}(0)=\boldsymbol{o}\right.$ and $\left.\boldsymbol{\boldsymbol { r }}(L)=L_{1} \boldsymbol{e}_{1}+L_{2} \boldsymbol{e}_{2}+L_{3} \boldsymbol{e}_{3}\right\}$ and $\mathbb{V}_{0}=\left\{\delta \breve{\boldsymbol{r}} \in H^{1} \mid \delta \breve{\boldsymbol{r}}(0)=\boldsymbol{o}\right.$ and $\left.\delta \breve{\boldsymbol{r}}(L)=\boldsymbol{o}\right\}$. The variational problem associated with equation (9) is formulated as follows

Find $\breve{\boldsymbol{r}} \in \mathbb{V}$ such that for every $\delta \breve{\boldsymbol{r}} \in \mathbb{V}_{0}$

$$
\begin{aligned}
& \int_{0}^{L}\left(\breve{\boldsymbol{n}} \cdot \delta \breve{\boldsymbol{r}}_{s}\right) d s-\int_{0}^{L}(\breve{\boldsymbol{f}} \cdot \delta \breve{\boldsymbol{r}}) d s=0 \\
& \breve{\boldsymbol{n}}=E A \breve{\Delta} \breve{\boldsymbol{r}}_{s} \text { for } s \in[0, L]
\end{aligned}
$$

This problem is a non quadratic minimization problem

$$
\begin{aligned}
& \text { Find } \breve{\boldsymbol{r}} \in \mathbb{V} \text { minimizing } \\
& J(\breve{\boldsymbol{r}})=\int_{0}^{L}\left(\frac{1}{2} E A \breve{\Delta}^{2}-\breve{\boldsymbol{r}} \cdot \breve{\boldsymbol{f}}\right) d s
\end{aligned}
$$

\section{Mixed variational formulation of the static problem}

The static problem can be solved using the displacement-only formulation (10) given above. For data assimilation purposes, however, it is interesting to introduce the strain as an unknown by imposing its definition as a constraint. More precisely, the relation $\breve{\Delta}=1 / 2\left(\breve{\boldsymbol{r}}_{s} \cdot \breve{\boldsymbol{r}}_{s}-1\right)$ in equation (9) is relaxed and 
considered as a constraint on $(\breve{r}, \breve{\Delta})$. Among the several options to impose a constraint in an optimization problem, we choose the method of Lagrange multipliers. Letting $\breve{T}$ be the Lagrange multiplier associated with the constraint, the problem (10) reads

Find $\breve{\boldsymbol{r}} \in \mathbb{V}, \breve{\Delta} \in \mathbb{P}_{\Delta}$ and $\breve{T} \in \mathbb{P}_{T}$

such that $(\breve{\boldsymbol{r}}, \breve{\Delta}, \breve{T})$ is a saddle point of

$J(\breve{\boldsymbol{r}}, \breve{\Delta}, \breve{T})=\int_{0}^{L}\left[\frac{1}{2} E A \breve{\Delta}^{2}-\breve{T}\left(\breve{\Delta}-\breve{\Delta}^{e}\right)-\breve{\boldsymbol{r}} \cdot \breve{\boldsymbol{f}}\right] d s$

where $\breve{\Delta}^{e}:=\frac{1}{2}\left(\breve{\boldsymbol{r}}_{s} \cdot \breve{\boldsymbol{r}}_{s}-1\right)$

By saddle point we mean that such $J(\breve{r}, \breve{\Delta}, \breve{T})$ is a relative minimum for $\breve{\boldsymbol{r}}$ and $\breve{\Delta}$ and a relative maximum for $\breve{T}$. We recall that the boundary conditions are taken into account within appropriate spaces $\mathbb{V}, \mathbb{P}_{\Delta}$, and $\mathbb{P}_{T}$.

Let us briefly show how equation (11) is related to the classical formulation (9). For any saddle point $(\breve{\boldsymbol{r}}, \breve{\Delta}, \breve{T})$ of the function $J$ in equation (11), the first variation $\delta J(\breve{\boldsymbol{r}}, \breve{\Delta}, \breve{T})$ vanishes, that is

$$
\begin{aligned}
\delta J= & \int_{0}^{L}\left[(E A \breve{\Delta}-\breve{T}) \delta \breve{\Delta}+\breve{T} \breve{\boldsymbol{r}}_{s} \cdot \delta \breve{\boldsymbol{r}}_{s}\right. \\
& \left.-\left(\breve{\Delta}-\breve{\Delta}^{e}\right) \delta \breve{T}-\breve{\boldsymbol{f}} \cdot \delta \breve{\boldsymbol{r}}\right] d s=0
\end{aligned}
$$

In equation (12), we recognize the following three sets of equations:

- $E A \breve{\Delta}=\breve{T}$ is the constitutive equation. As one expects, the Lagrange multiplier associated with the constraint is the tension $\widetilde{T}$.

- $\breve{\Delta}=\breve{\Delta}^{e}$ is the strain-displacement relationship, which plays the role of a constraint in equation (11).

- After an integration by parts on the integral $\int_{0}^{L}\left(\widetilde{T} \breve{\boldsymbol{r}}_{s} \cdot \delta \breve{\boldsymbol{r}}_{s}-\boldsymbol{f} \cdot \delta \breve{r}\right) d s$, we recover the strong form of the static equilibrium $\left(\widetilde{T} \breve{\boldsymbol{r}}_{s}\right)_{s}+\breve{\boldsymbol{f}}=0$.

Starting form equation (10), $J(\breve{\boldsymbol{r}}, \breve{\Delta}, \breve{T})$ is constructed as a $\mathrm{Hu}$-Washizu functional for the cable problem. More information on the $\mathrm{Hu}-$ Washizu formulation can be found in $\mathrm{He}^{22}$ Additional details on the numerical implementation of equation (12) are reported in Appendix 1.

It is convenient to introduce the quantities

$$
\kappa=E A / E A_{\text {th }} \quad m=10^{6} \rho \mathrm{Al} / E A_{\text {th }}
$$

where $l=\sqrt{L_{1}^{2}+L_{2}^{2}+L_{3}^{2}}$ and $E A_{t h}=3 / 16 E \pi d_{c}^{2}$ with $E$ being an estimate of the Young's modulus of the wires in the cable and $d_{c}$ being its diameter. The parameter $\kappa$ is nondimensional and $m^{-1}$ has the dimension of an acceleration. By employing SI units, $\kappa$ and $m$ are both close to unity for most cables commonly used in civil engineering. In the following, our parameters of interest will be $\kappa, m$, and $L$. Note that according to equation (13), $\kappa$ and $m$ are directly related to the axial stiffness and the mass per unit length, respectively.

\section{Data misfit}

\section{Setting the stage: why a standard residual does not work?}

We suppose that the monitoring system consists of $N$ strain gauges and $M$ displacement sensors. Our goal is to recover the tension of the cable or the corresponding safety factor $\gamma$ defined by equation (8) in the static configuration. We let $a$ be the natural static configuration of the cable laying under its own weight.

The most natural choice for $\mathfrak{J}$ is

$$
\begin{aligned}
\mathfrak{J}^{a}(L, \kappa, m) & =\sum_{i=1}^{N} \frac{c_{\text {str }}}{2}\left(\breve{\Delta}^{i}-\Delta^{i}\right)^{2} \\
& +\sum_{j=1}^{M} \frac{c_{\text {disp }}}{2}\left(\stackrel{\leftrightarrow}{r}_{1}^{j}-r_{1}^{j}\right)^{2}
\end{aligned}
$$

where the superscripts $i$ and $j$ are the sensor numbers, $r_{1}$ is the vertical component of the position vector $r$, and $\Delta$ is the axial strain. Breve quantities $\left(\breve{r}_{1}, \breve{\Delta}\right)$ are model-based, and non-breve quantities $\left(r_{1}, \Delta\right)$ denote the experimental data. The weighting parameters $c_{s t r}$ and $c_{d i s p}$ in equation (14) need to be chosen so that (1) accuracies of the sensors are respected and (2) the two resulting quantities are of the same order of magnitude. The data misfit functional equation (14) proves very difficult to handle in practice. First, absolute strain measures require the placement of sensors to be done in a stress-free configuration of the cable, which is rarely the case. Moreover, when taut cables are considered, a small bias on its sag characterization can lead to large errors in terms of tension identification. More importantly, even when the natural length of the cable $L$ is known, the minimization problem $\min _{(\kappa, m)} \mathfrak{J}^{a}(L, \kappa, m)$ is strongly ill-conditioned for the multiplicity of solutions and the non-uniqueness of the corresponding tensions found in the cable. To illustrate this point, we consider the example of $21.5-\mathrm{m}$ long cable tensioned with $\gamma=2.3$ and equipped with three strain sensors (labeled as JI, JII, and JIII) and two displacement sensors (labeled as D1 and D2). This specific configuration will be further discussed in the experimental validation part of this article. 


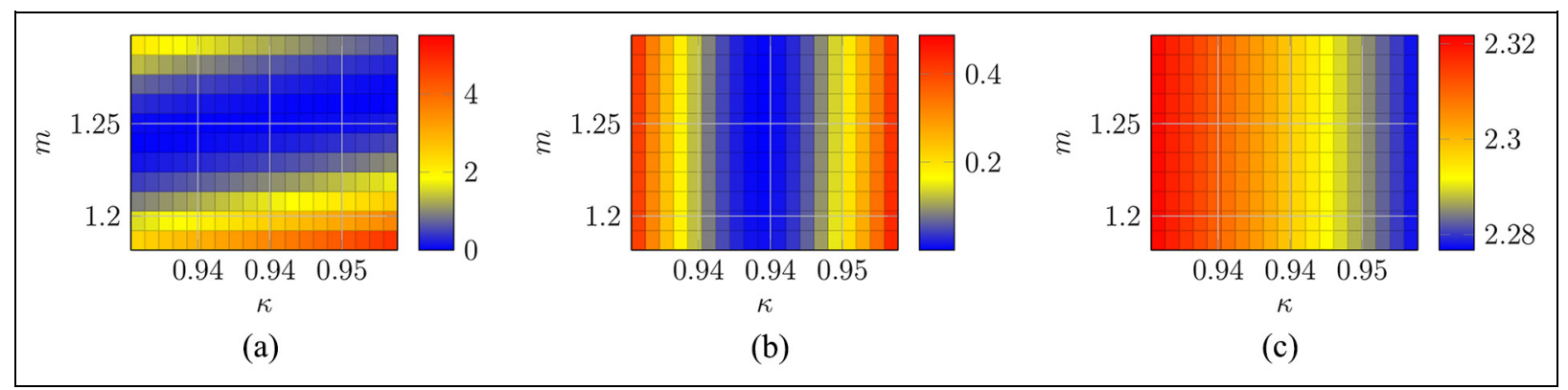

Figure 2. (a) Values of $\mathfrak{J}^{a}(L, \kappa, m)$ when configuration $a$ only is considered, (b) values of the proposed data misfit functional $\mathfrak{J}^{\mathfrak{a}, \mathfrak{b}}(L, \kappa, m)$ based on the difference between configurations $a$ and $b$, and (c) corresponding safety factors. The cable has been simulated with $L=21.5 \mathrm{~m}, \kappa=9.44410^{-1}$, and $m=1.253$.

The function $(\kappa, m) \mapsto \mathfrak{J}^{a}(L, \kappa, m)$ is shown in Figure 2(a) using simulated data $\left(r_{1}, \Delta\right)$ obtained from the solution of the static problem (10) with $\kappa=9.44410^{-1}$ and $m=1.253$. Weighting parameters $c_{s t r}$ and $c_{d i s p}$ are equal to $10^{12}$ and $10^{4} / d_{c}^{2}$, respectively, where $d_{c}$ is the diameter of the cable. As one can observe in Figure 2(a), the function $(\kappa, m) \mapsto \mathfrak{J}^{a}(L, \kappa, m)$ features several minima (indicated as blue points). Figure 2(c) shows the value of the cable safety factor $\gamma$ as a function of $\{\kappa, m\}$. Comparing Figure 2(a) and (c) reveals that each couple $(\kappa, m)$ solving the minimization problem $\min _{(\kappa, m)} \mathfrak{J}^{a}(L, \kappa, m)$ leads to a different value of the cable safety factor (and of the tension). This was expected because one can only choose independently four among the five parameters of the cable $\left\{\kappa, m, \gamma, L, \mathbf{L}_{s}\right\}$, where $\mathbf{L}_{s}$ is the chord vector defined by the two cable supports. Note that the direction of $\mathbf{L}_{s}$ is irrelevant and $\mathbf{L}_{s}$ could be replaced either by the space coordinates of the cable supports or by the cable chord length $L_{s}=\left\|\mathbf{L}_{s}\right\|$ and the chord angle with respect to the horizontal plane. It is proposed to use a data misfit functional based on two configurations $a$ and $b$. The configuration $a$ is the natural static configuration of the cable laying under its own weight, as considered in equation (14). The configuration $b$ is the static configuration of the cable when subject to a given additional load. The data misfit functional $\mathfrak{J}^{\mathfrak{a}, \mathfrak{b}}(L, \kappa, m)$ that we consider is defined by

$$
\begin{gathered}
\mathfrak{J}^{\mathfrak{a}, \mathfrak{b}}=J_{s t r}+J_{\text {disp }} \\
J_{s t r}=\sum_{i=1}^{N} \frac{c_{\text {str }}}{2}\left[\left(\stackrel{\Delta}{ }^{i, b}-\stackrel{\Delta}{s}^{i, a}\right)-\left(\Delta^{i, b}-\Delta^{i, a}\right)\right]^{2} \\
J_{\text {disp }}=\sum_{j=1}^{M} \frac{c_{\text {disp }}}{2}\left[\left(\stackrel{r}{r}, b_{1}^{j, j} \vec{r}_{1}^{j, a}\right)-\left(r_{1}^{j, b}-r_{1}^{j, a}\right)\right]^{2}
\end{gathered}
$$

where the superscripts $a$ and $b$ denote the configuration, breve quantities are calculated via the static problem (11), and other quantities are the measured data.

To first illustrate the relevance of the data misfit functional in equation (17), consider the example of a 21.5-m cable introduced previously. In configuration $b$, the cable is subjected to a point load of $1 \mathrm{kN}$ as depicted in Figure 3. The corresponding function $(\kappa, m) \mapsto \mathfrak{J}^{\mathfrak{a}, \mathfrak{b}}(L, \kappa, m)$ is shown in Figure 2(b). As it can be seen in Figure 2(b) and (c), the modification of the data misfit does not prevent the ill-conditioning of the minimization problem $\min _{(\kappa, m)} \mathfrak{J}^{\mathfrak{a}, \mathfrak{b}}(L, \kappa, m)$ but it does lead to a unique and correct safety factor value $\gamma=2.3$. Looking at Figure 2(a) to (c), it can be anticipated that $\mathfrak{J}^{a}+\mathfrak{J}^{\mathfrak{a}, \mathfrak{b}}$ would lead to a well-posed problem and a correct identification of all cables' parameters, namely, the tension, the mass per unit length, and the axial stiffness. However, since it is easier to measure strain differences and displacements than absolute strains and positions, we choose to construct our tension assessment method using $\mathfrak{J}^{\mathfrak{a}, \mathfrak{b}}$ only. As it will be developed in the following sections, the proposed data misfit can be considered as a reliable workaround for the tension assessment problem.

\section{Numerical exploration of the novel data misfit functional $\mathfrak{J}^{\mathfrak{a}, \mathfrak{b}}$}

In this section, we use some numerical simulations to further highlight the most striking feature of the data misfit functional $\mathfrak{J}^{\mathfrak{a}, \mathfrak{b}}$ in equation (15), namely, its ability to convey the right information in view of tension assessment. We choose to explore two completely different configurations of a generic cable - one taut and the other sagged - with a span of $50 \mathrm{~m}$ and a diameter $50 \mathrm{~mm}$ in both cases. The true values of the axial stiffness and linear mass of the cables are $\kappa_{t c}=1.0$ and $m_{t c}=2.297$. The natural length of the taut cable is 


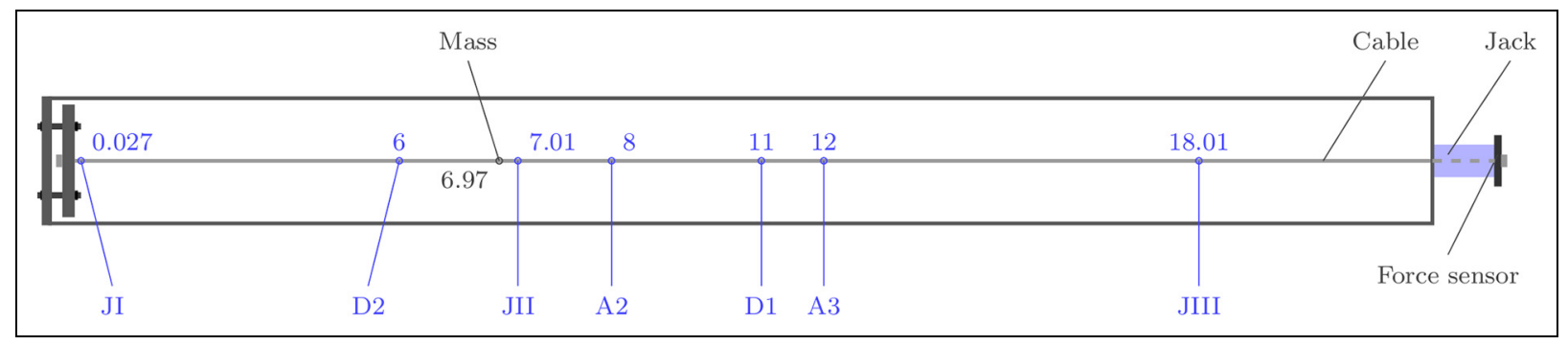

Figure 3. Drawing of the test setup used in the experimental validation campaign.

D: displacement sensor; A: accelerometer; J: strain gauges. Positioning is given in meters from the left anchorage.

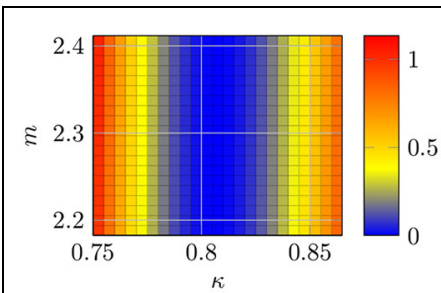

(1a)

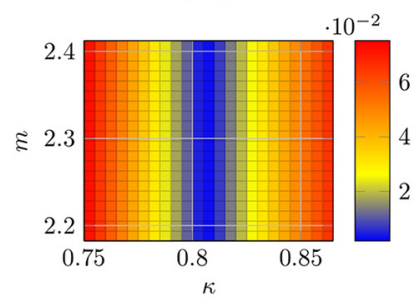

(2a)

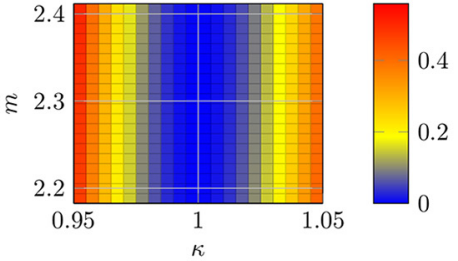

(1b)

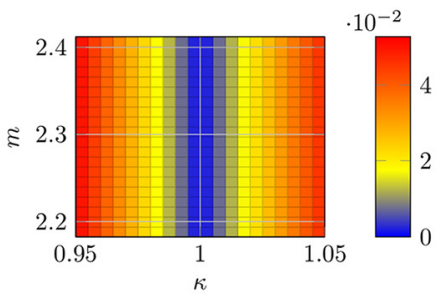

(2b)

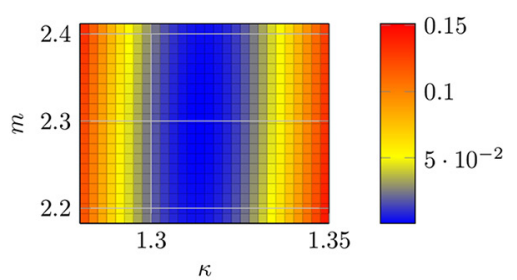

(1c)

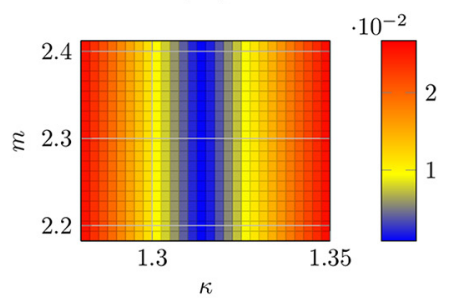

$(2 \mathrm{c})$

Figure 4. Taut cable: (I) parametric study of $\mathfrak{J}^{\mathfrak{a}, \mathfrak{b}}(L, \kappa, m)$ for $(\mathrm{Ia}) L=0.999 L_{t c}$, (Ib) $L=L_{t c}$, and (Ic) $L=I .00 I L_{t c}$ and $(2)$ corresponding safety factor errors $\left|\gamma-\gamma_{t c}\right| / \gamma_{t c}$, where $\gamma_{t c}=2.3$.

$L_{t c}=49.79 \mathrm{~m}$ and that of the sagged cable is $L_{t c}=57.5 \mathrm{~m}$. The taut and sagged cables have a sag-to-span ratio of $610^{-6}$ and 0.25 , respectively, and a safety factor of 2.3 and 550, respectively. Data measures are simulated via the static problem (10), and the weighting parameters $c_{s t r}$ and $c_{d i s p}$ are equal to $10^{12}$ and $10^{4} / d_{c}^{2}$, respectively, where $d_{c}$ is the diameter of the cable. These values are consistent with the accuracies of most strain gauges $(0.1 \mu \mathrm{def})$ and displacement sensors $(0.1 \mathrm{~mm})$. Because the exact length of the cable is an unknown of the problem, the numerical exploration of the data misfit $(\kappa, m) \mapsto \mathfrak{J}^{\mathfrak{a}, \mathfrak{b}}(L, \kappa, m)$ is done with three different lengths in both cases. For the taut cable, it is reasonable to choose three values of $L$ that are close to the actual length $L_{t c}$, and we therefore numerically investigate $(\kappa, m) \mapsto \mathfrak{J}^{\mathfrak{a}, \mathfrak{b}}(\kappa, m) \quad$ for $\quad L=0.999 L_{t c}, \quad L=L_{t c}, \quad$ and $L=1.001 L_{t c}$ as shown in Figure 4. For the sagged cable, we let the uncertainties on the length of the cable be greater by choosing $L=0.99 L_{t c}, L=L_{t c}$, and $L=1.01 L_{t c}$ as shown in Figure 5. Observing the two sets of graphs raises three main comments. First, the data misfit functional allows an exact assessment of the tension whenever the exact length of the cable is known as shown by the perfect alignment of the minimal values in graphs (b). Second, we notice that the problem is more sensitive to the stiffness parameter $\kappa$ for the taut cable and to the mass per unit length parameter $m$ for the sagged cable. It is not surprising since the displacements of a cable subject to an applied load are related to elastic deformation for a taut cable and to geometric adaptation (with little elastic deformation) for a sagged cable. This is also highlighted by the design of Figures 4 and 5 that uses inverted $\kappa$ - and $m$-axes but results in similar graphs. Considering both parameters $\kappa$ and $m$ as unknowns of our inverse problem ensures its universal applicability. This feature will be illustrated in the following section. Last but not least, the error introduced by the lack of precision on the length of the cable propagates to the tension assessment but has a very limited impact. Even in the worse cases depicted in graphs (1a), 


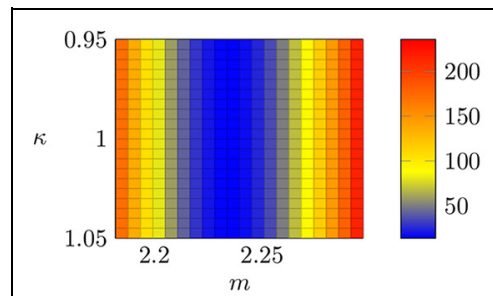

(1a)

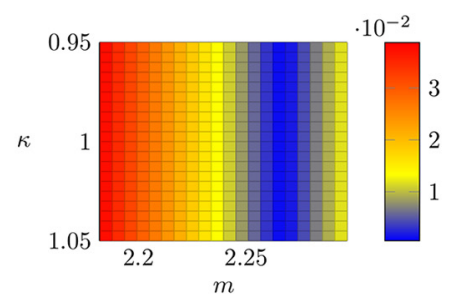

(2a)

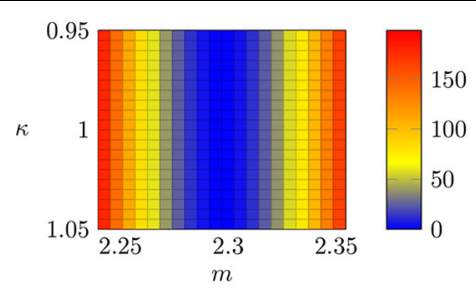

(1b)

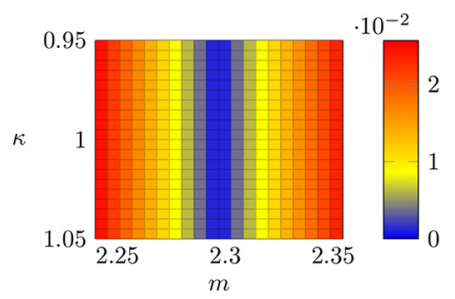

$(2 b)$

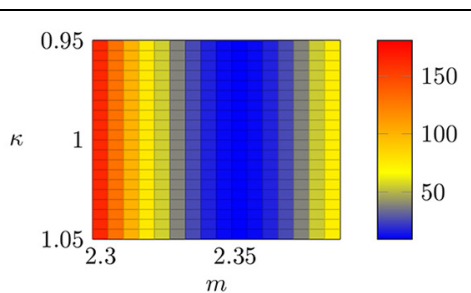

(1c)

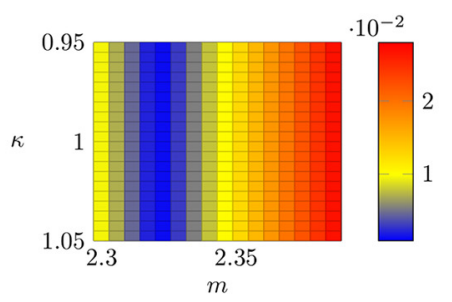

(2c)

Figure 5. Sagged cable: (I) parametric study of $\mathfrak{J}^{\mathfrak{a}, \mathfrak{b}}(L, \kappa, m)$ for $(\mathrm{Ia}) L=0.99 L_{t c}$, (Ib) $L=L_{t c}$, and (Ic) $L=I .0 I L_{t c}$ and $(2)$ corresponding safety factor errors $\left|\gamma-\gamma_{t c}\right| / \gamma_{t c}$, where $\gamma_{t c}=550$.

(2a) and (1c), (2c) of Figure 5 for which a $1 \%$ error has been introduced on a very loose cable $\left(\gamma_{t c}=550\right)$, the data misfit functional minima lead to less than $1.5 \%$ error on the tension.

The minimization problem is ill-posed since it has multiple solutions in all the investigated cases (taut and sagged cables). However, these solutions lead to a correct identification of the tension in the cable. Parametric studies showed that the minimization problem is very sensitive to the length parameter $L$ in all cases and sensitive to the stiffness parameter $\kappa$ or to the mass per unit length parameter $m$ when the cable is taut or sagged, respectively.

A flow chart of the proposed method construction is shown in Figure 6. Conclusions of the numerical investigations made for $\mathfrak{J}^{a}$ in section "Setting the stage: why a standard residual does not work?" and the ones presented for $\mathfrak{J}^{a, b}$ are summarized. On the flow chart, it can clearly be seen that both data misfits are based on the nonlinear elastic model of cable proposed in section "Cable model," thus covering all types of cables. Although the ill-conditioning exists for both data misfits, $\mathfrak{J}^{a, b}$ is the only one leading to a correct tension assessment.

\section{Inverse problem}

\section{Identification of the natural length of the cable}

As confirmed by the numerical investigations presented in section "Data misfit," the static behavior of taut cables is more sensitive to variations of $\kappa$ and $L$ while for sagged cables, the two parameters $m$ and $L$ have a preponderant part.

In our work, all three parameters $\{\kappa, m, L\}$ are treated as unknown since the tension assessment method covers all types of cables (taut, sagged, and nonshallow). However, looking for the triplet $\{\kappa, m, L\}$ in a single-step procedure would lead to an illconditioned problem showing slow convergence due to endless updates. Because of its importance in taut and sagged cables, we choose to address first the identification of the natural length $L$ for given $\kappa$ and $m$. Then, we identify parameters $\kappa$ and $m$ for a fixed length $L$. In the following, we illustrate the suitability of this choice with three examples: a taut cable and a sagged cable as described in section "Numerical exploration of the novel data misfit functional $\mathfrak{J}^{\mathfrak{a}, \mathfrak{b}}$ " and an intermediate one for which $\gamma=29.13$. For each of the three cables, we show a numerical exploration of $\mathfrak{J}^{\mathfrak{a}, \mathfrak{b}}$ as a function of $(\kappa, L)$ keeping $m$ constant and as a function of $(m, L)$ keeping $\kappa$ constant. The ensuing graphs are reported in Figures 7 and 8, respectively, together with their safety factor errors. Here it is to be pointed out that in our simulations, sagged cables correspond to large safety factors $\gamma$ but one could also have highly tensioned sagged cables. Tensioned and sagged cables are notably used for suspension bridges with sag-to-span ratios close to 0.125 and safety factors between approximately 2.3 and 7.

Looking at the four graphs (a) in Figures 7 and 8, we see the data misfit functional main variations for taut cables. In Figure 7(1a) and (2a), we notice that $\mathfrak{J}^{\mathfrak{a}, \mathfrak{b}}$ and the relative error made on $\gamma_{t c}$ have similar 


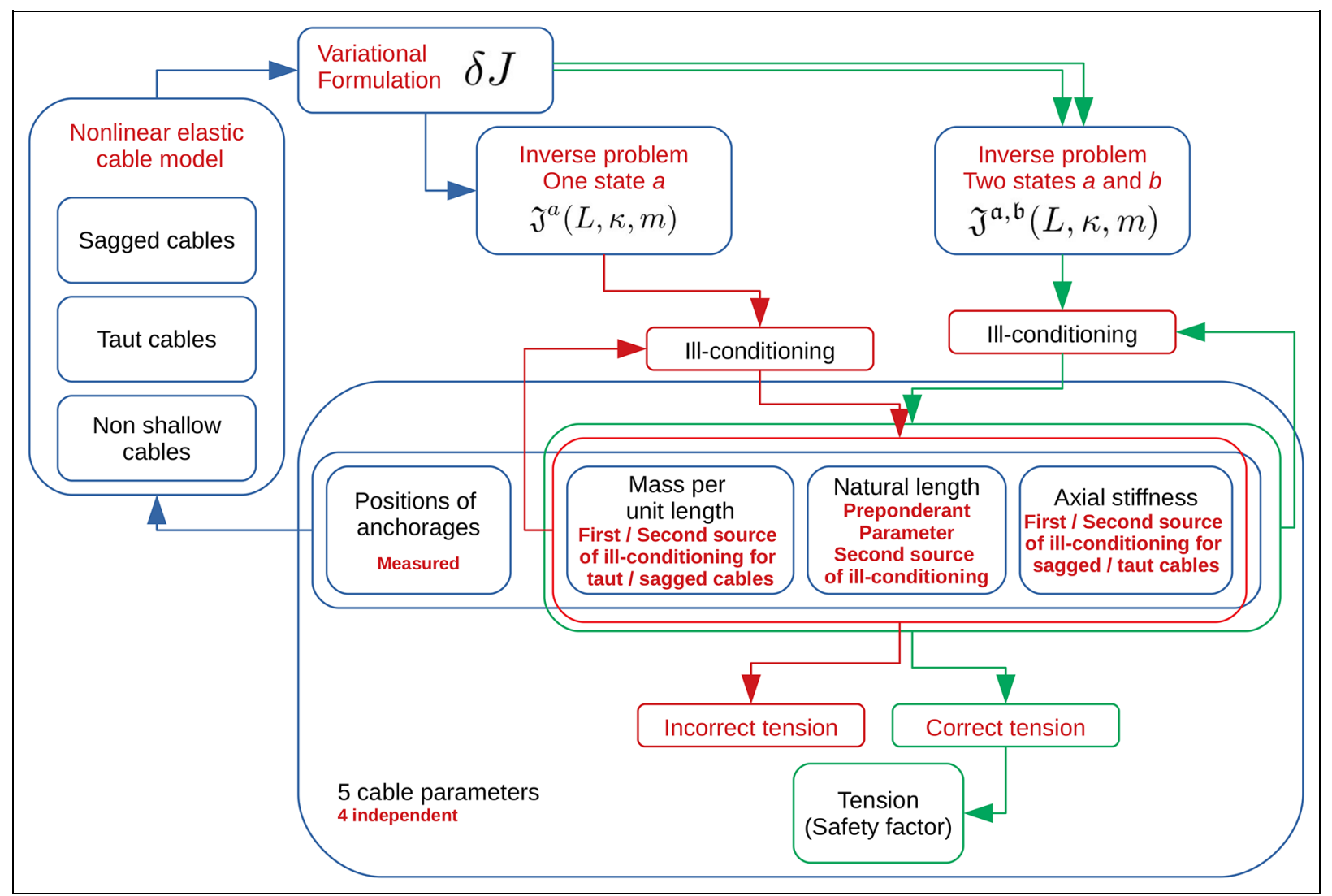

Figure 6. Flow chart of the proposed method. The tension assessment uses two configurations $a$ and $b$ introduced in a novel data misfit $\mathfrak{J}^{a, b}$ via an original variational formulation $\delta J$ of a nonlinear elastic model covering all types of cables. Sources of ill-conditioning are highlighted and the more conventional data misfit $\mathfrak{J}^{a}$ leading to an incorrect tension assessment is reported for comparison purposes.

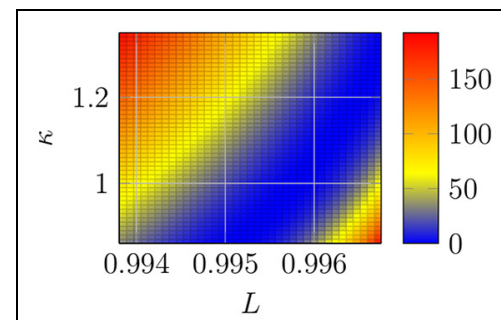

(1a)

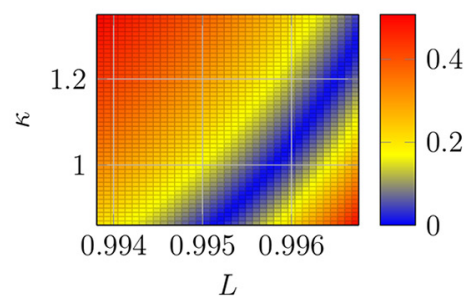

(2a)

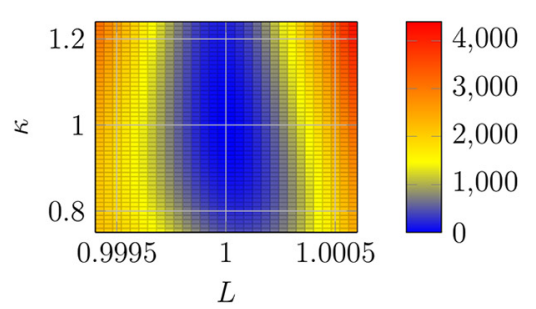

(1b)

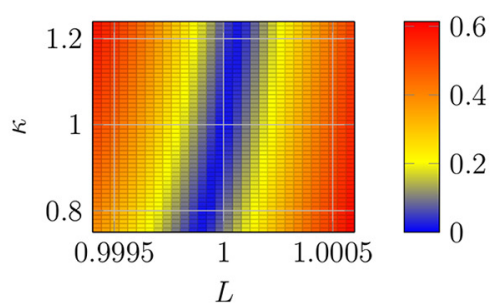

(2b)

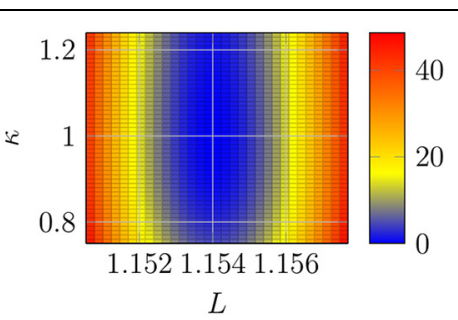

(1c)

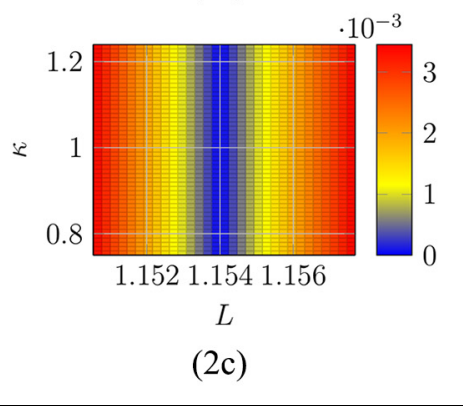

Figure 7. (I) Parametric study of $\mathfrak{J}^{\mathfrak{a}, \mathfrak{b}}(L, \kappa)$ with $m$ fixed for (la) a taut cable with $\gamma=2.3$, (lb) an intermediate cable with $\gamma=29.13$, and (IC) a sagged cable with $\gamma=550$. (2) Corresponding safety factor errors $\left|\gamma-\gamma_{t c}\right| / \gamma_{t c}$. The graphs illustrate the predominance of the length parameter in the sagged case while the taut cable case is also impacted by the stiffness parameter. 


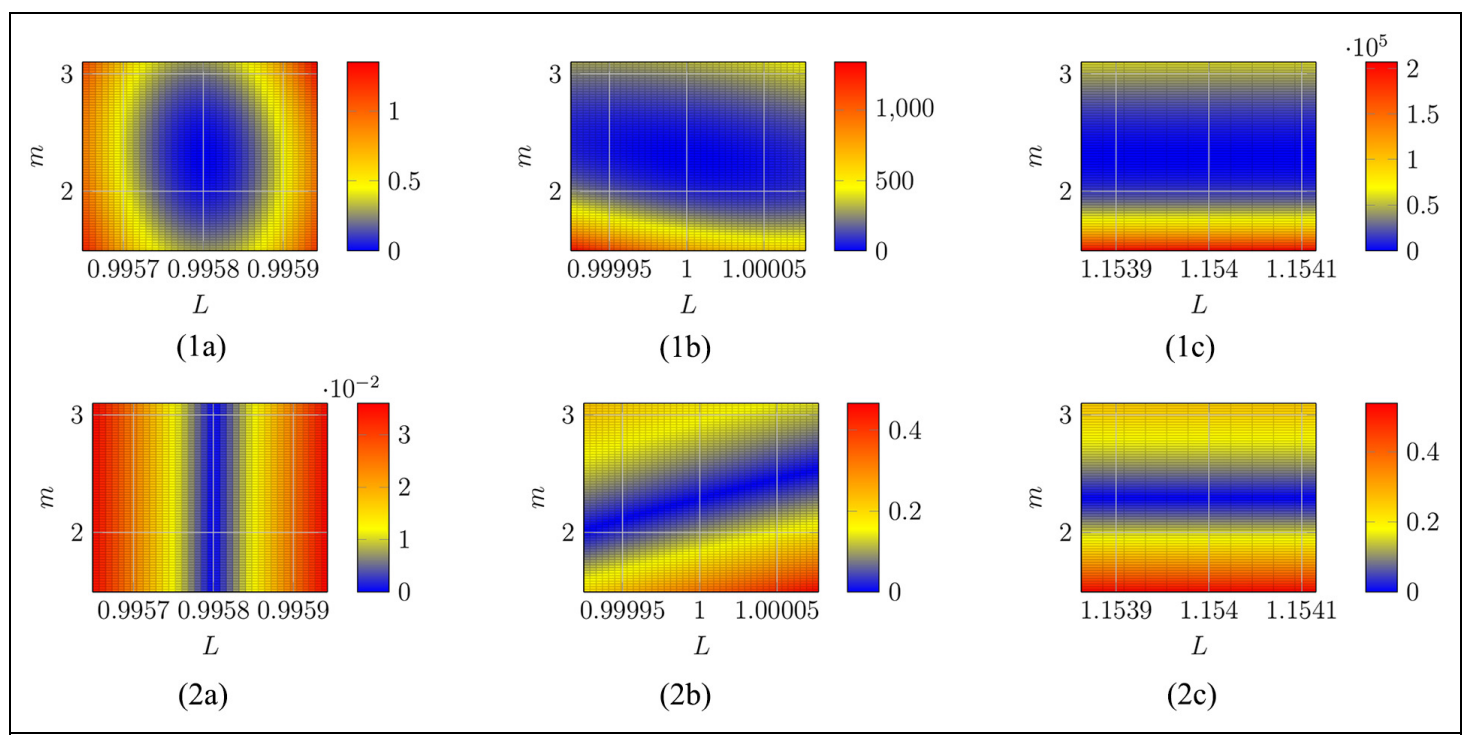

Figure 8. (I) Parametric study of $\mathfrak{J}^{\mathfrak{a}, \mathfrak{b}}(L, m)$ with $\kappa$ fixed for (la) a taut cable with $\gamma=2.3$, (Ib) an intermediate cable with $\gamma=29.13$, and (Ic) a sagged cable with $\gamma=550$. (2) Corresponding safety factor errors $\left|\gamma-\gamma_{t c}\right| / \gamma_{t c}$. For the taut cable, the data misfit is highly sensitive to the length parameter and could theoretically lead to a correct identification of $m$. In the sagged case, the parameter $m$ is predominant.

topologies and they are minimal for the same values of $(\kappa, L)$. It shows that the data misfit is quite a good choice for tension identification. Figure 7(2a) has a simple physical interpretation: if $m$ can be neglected and $\mathbf{L}_{s}$ is fixed, two cables have the same tension as long as their difference in length is compensated by their difference in stiffness. Using the data misfit in this specific case is very efficient since one can either fix $\{\kappa, m\}$ and search for $L$ such that $\mathfrak{J}^{\mathfrak{a}, \mathfrak{b}}(L)$ reaches its minimum or fix $\{L, m\}$ and search $\kappa$ such that $\mathfrak{J}^{\mathfrak{a}, \mathfrak{b}}(\kappa)$ reaches its minimum. In both cases, the problem of the tension assessment is well-posed and leads to the correct tension. In turn, Figure 8(2a) confirms the limited influence of $m$ while Figure 8(1a) suggests that the data misfit is able to return a correct value of $m$.

For sagged cables, the four graphs (c) in Figures 7 and 8 show the clear preponderance of the parameter $m$. This was expected since for cables with very large sag, the maximum tension of the cable depends more directly on the total weight it carries, that is, the product $m L$. The error made on the tension assessment will therefore be roughly proportional to $d(m L) / m L=$ $d m / m+d L / L$ with $d m / m \gg d L / L$. This is illustrated in Figure 7(1c) and (2c) when $m$ is fixed and in Figure $8(1 \mathrm{c})$ and (2c) when $\kappa$ is fixed.

The four graphs (b) in Figures 7 and 8 are not as easy to interpret as in the previous cases since they illustrate a cable that is neither taut nor completely sagged. For this cable configuration and looking at the graphs, it is reasonable to think that all parameters are of importance, with a clear preponderance for the cable length.

In accordance with these three last numerical investigations, we choose to address first the identification of the natural length $L$ at fixed $\kappa$ and $m$ and then the identification of parameters $\kappa$ and $m$ at fixed length $L$. Length search is done in a very simple way via a line search method, making use of the static problem (10) to numerically assess the derivative of $\mathfrak{J}^{\mathfrak{a}, \mathfrak{b}}$ with respect to $L$. The corresponding pseudocode is given in Algorithm 1 in Appendix 1.

\section{Identification of the tension}

Let $L$ be the length of the cable found via the line search method. In order to identify the tension, we need to find a set of parameters $(\kappa, m)$ that minimizes $\mathfrak{J}^{\mathfrak{a}, \mathfrak{b}}(L, \kappa, m)$. Standard minimization algorithms require the evaluation of the sensitivities $\partial \mathfrak{J}^{\mathfrak{a}, \mathfrak{b}} / \partial \kappa$ and $\partial \mathfrak{J}^{\mathfrak{a}, \mathfrak{b}} / \partial m$ at each iteration. In our case, evaluating those sensitivities is not straightforward since the function $\mathfrak{J}$ in equation (17) is not expressed explicitly in terms of $(\kappa, m)$. Rather, the function $\mathfrak{J}$ depends implicitly on $(\kappa, m)$ through the influence of those parameters on the static solution $\left(r^{i, a}, \vec{r}^{i, b}, \breve{\Delta}^{i, a}, \vec{\Delta}^{i, b}\right)$. That difficulty can be overcome using the adjoint state method, as is now explained (a complete introduction on inverse problems can be found in $\mathrm{Bui}^{18}$ ). The data misfit functional is rewritten as 


$$
\begin{gathered}
J_{s t r}=\sum_{i=1}^{N} \frac{c_{s t r}}{2}\left[\int_{0}^{L} d \breve{\Delta}^{i, b, a} \psi^{i}-d \Delta^{i, b, a}\right]^{2} \\
J_{\text {disp }}=\sum_{j=1}^{M} \frac{c_{\text {disp }}}{2}\left[\int_{0}^{L} d r_{1}^{j, b, a} \psi^{j} d s-d r_{1}^{j, b, a}\right]^{2}
\end{gathered}
$$

where $d \Delta^{i, b, a}=\breve{\Delta}^{i, b}-\breve{\Delta}^{i, a}, d \Delta^{i, b, a}, d \dot{r}_{1}^{j, b, a}, d r_{1}^{j, b, a}$ have similar expressions and $\psi^{i}(s)=n / L \operatorname{Dirac}\left(s-s^{i}\right)$, and $n$ is the number of mesh elements. Define the Lagrangian $\mathfrak{L}^{a, b}$ as

$$
\mathfrak{L}^{a, b}=\mathfrak{J}^{\mathfrak{a}, \mathfrak{b}}-J^{b}-J^{a}
$$

where

$$
\begin{aligned}
J^{b} & =\int_{0}^{L}\left[\left(\kappa \breve{\Delta}^{b}-\breve{T}^{b}\right) \breve{\Delta}^{* b}+\breve{T}^{b} \breve{\boldsymbol{r}}_{s}^{b} \cdot \breve{\boldsymbol{r}}_{s}^{* b}\right. \\
& \left.-\left(\breve{\Delta}^{b}-\breve{\Delta}^{e, b}\right) \breve{T}^{* b}-\breve{\boldsymbol{f}}^{b} \cdot \breve{\boldsymbol{r}}^{* b}\right] d s
\end{aligned}
$$

and $J^{a}$ has the same expression with $a$ superscripts instead of $b$. We recall that $\Delta:=1 / 2\left(\breve{\boldsymbol{r}}_{s} \cdot \breve{\boldsymbol{r}}_{s}-1\right)$. The Lagrangian equation (20) is formula (4) applied to the special data misfit $\mathfrak{J}^{\mathfrak{a}, \mathfrak{b}}$. The direct variational problem is obtained via $\mathfrak{L}^{a, b}$ derivatives with respect to starred quantities (dual variables)

$$
\begin{aligned}
\delta J^{b}= & \int_{0}^{L}\left[\left(\kappa \breve{\Delta}^{b}-\breve{T}^{b}\right) \delta \breve{\Delta}^{* b}+\breve{T}^{b} \breve{\boldsymbol{r}}_{s}^{b} \cdot \delta \breve{\boldsymbol{r}}_{s}^{* b}\right. \\
& \left.-\left(\breve{\Delta}^{b}-\breve{\Delta}^{e, b}\right) \delta \breve{T}^{* b}-\breve{\boldsymbol{f}}^{b} \cdot \delta \breve{\boldsymbol{r}}^{* b}\right] d s=0
\end{aligned}
$$

A similar expression holds for $\delta J^{a}$. The sum $\delta J^{a}+\delta J^{b}$ is formula (5), where the two states $a$ and $b$ are used. Another way to obtain the direct variational problem is to imagine a non-physical state $a+b$ and use the variational formulation (12) to express $\delta J^{b}+\delta J^{a}$. An integration by parts of equation (22) is reported in Appendix 1 and leads to the strong form of the direct problem in $b$

$$
\left\{\begin{array}{l}
\left(\breve{T}^{b} \breve{\boldsymbol{r}}_{s}^{b}\right)_{s}+\breve{\boldsymbol{f}}^{b}=\boldsymbol{o} \\
\breve{T}^{b}=\kappa \breve{\Delta}^{b} \\
\breve{\Delta}^{b}=\vec{e}^{b} \\
\breve{\boldsymbol{r}}(0)=\boldsymbol{o}, \breve{\boldsymbol{r}}(L)=L_{1} \boldsymbol{e}_{1}+L_{2} \boldsymbol{e}_{2}+L_{3} \boldsymbol{e}_{3}
\end{array}\right.
$$

Same equations with $a$ superscripts complete the direct problem. Problem (23) corresponds to the static problem (9) for the configuration $b$. The adjoint problem is obtained computing the derivatives of $\mathfrak{L}^{a, b}$ with respect to $\left(r^{i, a}, r^{i, b}, \breve{\Delta}^{i, a}, \breve{\Delta}^{i, b}\right)$ (primal variables), giving

$$
\begin{aligned}
& \int_{0}^{L}\left(f^{\Delta} \delta \breve{\Delta}^{b}+\boldsymbol{f}^{r} \cdot \delta \boldsymbol{r}^{b}\right) d s \\
& =\int_{0}^{L}\left[\left(\kappa \breve{\Delta}^{* b}-\breve{T}^{* b}\right) \delta \breve{\Delta}^{b}\right. \\
& +\left(\breve{\boldsymbol{r}}_{s}^{b} \cdot \breve{\boldsymbol{r}}_{s}^{* b}-\breve{\Delta}^{* b}\right) \delta \breve{T}^{b} \\
& \left.+\left(\breve{T}^{b} \breve{\boldsymbol{r}}_{s}^{* b}+\breve{T}^{* b} \breve{\boldsymbol{r}}_{s}^{b}\right) \cdot \delta \breve{\boldsymbol{r}}_{s}^{b}\right] d s
\end{aligned}
$$

where $f^{\Delta}$ and $\boldsymbol{f}^{r}$ are the following quantities

$$
\begin{aligned}
f^{\Delta} & =-\sum_{i=1}^{N} c_{s t r}\left(\int_{0}^{L} d \Delta^{i, b, a} \psi^{i} d s-d \Delta^{i, b, a}\right) \psi^{i} \\
\boldsymbol{f}^{r} & =-\sum_{j=1}^{M} c_{d i s p}\left(\int_{0}^{L} d r_{1}^{j, b, a} \psi^{j} d s-r_{1}^{j, b, a}\right) \psi^{j} \boldsymbol{e}_{1}
\end{aligned}
$$

After an integration by parts (see Appendix 1), strong forms of the adjoint problem in $b$ can be recovered

$$
\left\{\begin{array}{l}
\left(\breve{T}^{b} \breve{\boldsymbol{r}}_{s}^{* b}+\breve{T}^{* b} \breve{\boldsymbol{r}}_{s}^{b}\right)_{s}+\boldsymbol{f}^{r}=\boldsymbol{o} \\
\breve{T}^{* b}=\kappa \breve{\Delta}^{* b}-f^{\Delta} \\
\breve{\Delta}^{* b}=\breve{\boldsymbol{r}}_{s}^{b} \cdot \breve{\boldsymbol{r}}_{s}^{* b} \\
\breve{\boldsymbol{r}}^{* b}(0)=\breve{\boldsymbol{r}}^{b}(0), \breve{\boldsymbol{r}}^{* b}(L)=\breve{\boldsymbol{r}}^{b}(L)
\end{array}\right.
$$

Same equations with $a$ superscripts complete the adjoint problem. Comparing equation (25) with equation (23), we notice the extra terms show up in the equilibrium equations and in the constitutive law compared to the direct problem. Moreover, it can be observed from the strain-displacement relationships and the equilibrium equations in equation (25) that the adjoint problem has the same mathematical structure as the linearized direct problem around its equilibrium. Sensitivities are obtained computing the derivatives of $\mathfrak{L}^{a, b}$ with respect to $m$ and $\kappa$

$$
\begin{aligned}
& \frac{\partial \mathfrak{J}^{\mathfrak{a}, \mathfrak{b}}}{\partial \kappa}=\frac{\partial \mathfrak{L}^{a, b}}{\partial \kappa}=-\int_{0}^{L}\left(\breve{\Delta}^{b} \breve{\Delta}^{* b}+\breve{\Delta}^{a} \breve{\Delta}^{* a}\right) d s \\
& \frac{\partial \mathfrak{J}^{\mathfrak{a}, \mathfrak{b}}}{\partial m}=\frac{\partial \mathfrak{L}^{a, b}}{\partial m}=-\int_{0}^{L} 10^{-6} g e_{1} \cdot\left(\breve{\boldsymbol{r}}^{* b}+\breve{\boldsymbol{r}}^{* a}\right) d s
\end{aligned}
$$

The above expressions show that the solutions of the direct and adjoint problems give a direct access to the sensitivities. This procedure can be embedded in any 


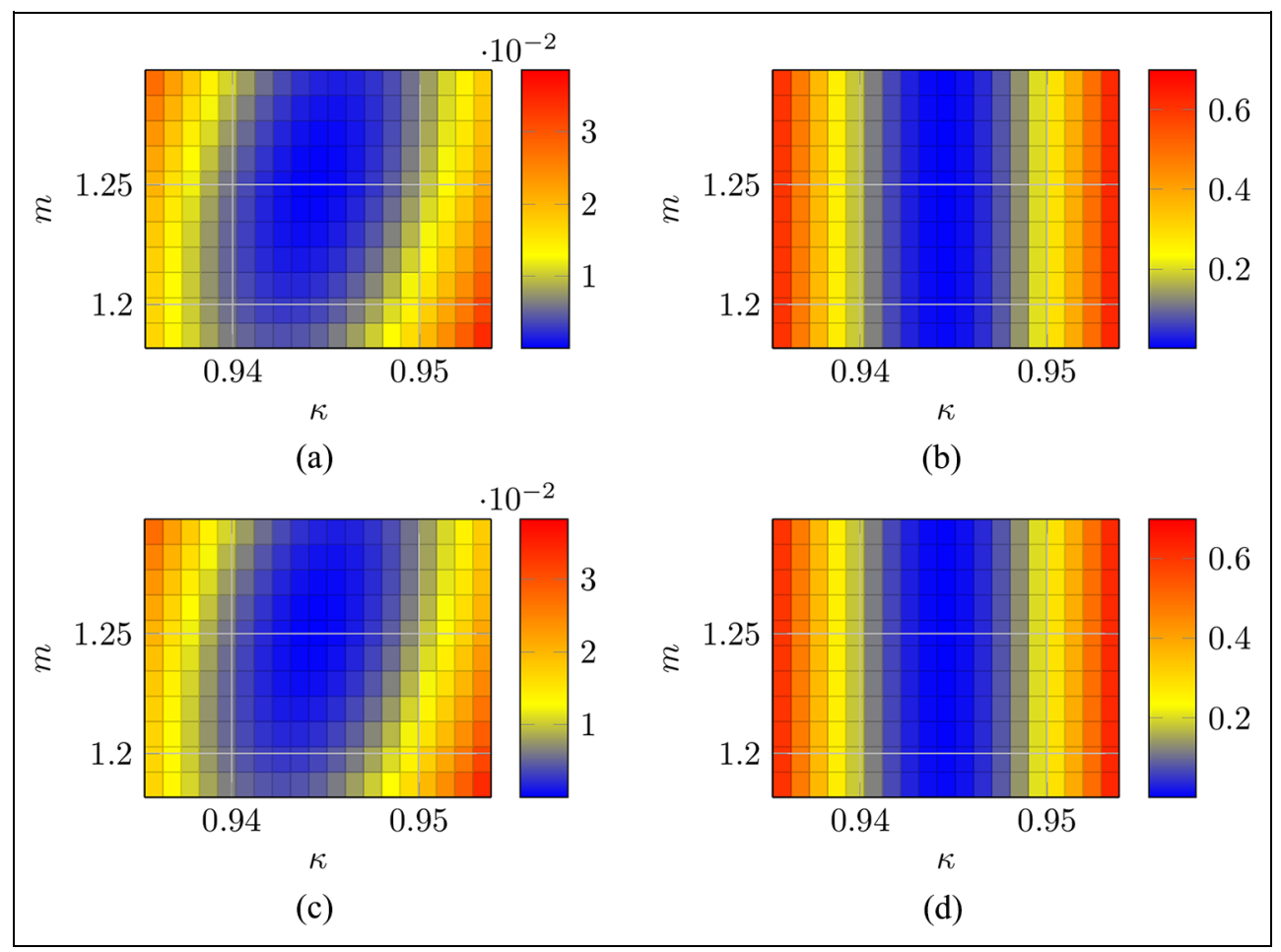

Figure 9. Data misfit values using one displacement sensor and one strain sensor in various positions: (a) displacement and strain sensors near the left anchorage at $s=1.75 \mathrm{~m}$ and $s=2.1 \mathrm{~m}$, respectively, (b) $s=10.5 \mathrm{~m}$ and $s=2.15 \mathrm{~m}$, respectively, (c) $s=1.75 \mathrm{~m}$ and $s=10.5 \mathrm{~m}$, respectively, and (d) $s=10.5 \mathrm{~m}$ and $s=10.5 \mathrm{~m}$, respectively. The $1.0 \mathrm{kN}$ point load is located at midspan in all four cases.

first-order optimization algorithm to seek the parameters $m$ and $\kappa$ minimizing the data misfit $\mathfrak{J}^{\mathfrak{a}, \mathfrak{b}}(L, \kappa, m)$. All the examples presented in this article have been obtained with the Barzilai-Borwein algorithm ${ }^{23}$ that can be found in Algorithm 2 in Appendix 1.

\section{Performance assessment of the inverse methodology}

\section{Sensor placement}

In Figure 9(a) to (d), a numerical exploration of $(\kappa, m) \mapsto \mathfrak{J}^{\mathfrak{a}, \mathfrak{b}}(L, \kappa, m)$ is presented for four typical sensor placements when the cable is subjected to a $1.0 \mathrm{kN}$ point load located at midspan. The cable considered is $21.5 \mathrm{~m}$ long with $\kappa_{t c}=0.944$ and $m_{t c}=1.253$. For this study, only one displacement sensor $(M=1)$ and one strain gauge $(N=1)$ are used. Data measures are simulated by solving the static problem with the true values $\left(\kappa_{t c}, m_{t c}\right)$. For each one of the two sensors, two positions are tested: one next to the left anchorage and the other at midspan, leading to four different combinations. As it can be seen with the chosen load magnitude for the point load, the minimization problem at hand shows two kinds of behavior: whenever the displacement sensor is placed near the anchorage, the problem seems to be well-posed and leads to the correct tension but would require strain and displacement sensors having extremely good precisions if compared to those usually used in bridge monitoring. On the contrary, when the displacement sensor is placed at midspan, the strain contribution is less visible and the problem is once again ill-posed but leads to the correct tension and can be conducted with a standard displacement sensor having a precision of $\pm 0.1 \mathrm{~mm}$.

\section{Load magnitude}

In Figure 10(a) to (c), a numerical investigation of $\mathfrak{J}^{\mathfrak{a}, \mathfrak{b}}(L, \kappa, m)$ is carried out at different load magnitudes. As in the previous section, only one displacement sensor and one strain gauge are used in the study. In order to test one of the most severe cases, we choose to place both sensors and the load point at $2.1 \mathrm{~m}$ from the left anchorage. Three tested load cases are presented: 1.0, 2.0 , and $3.0 \mathrm{kN}$. As expected, the three graphs have similar shapes (but different values) and lead to the correct tension identification but the load magnitude clearly acts as a regularization of the data misfit functional: in practice, a greater load will ease the tension identification. In fact, when the cable is tensed and the applied load is small, the relation between the load 


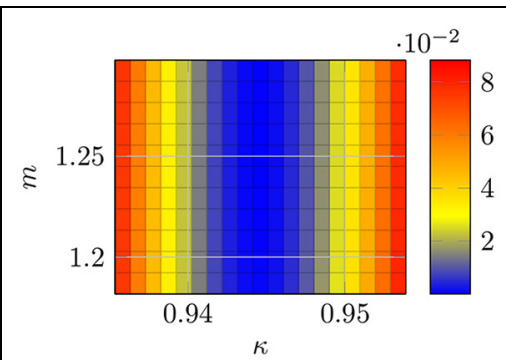

(a)

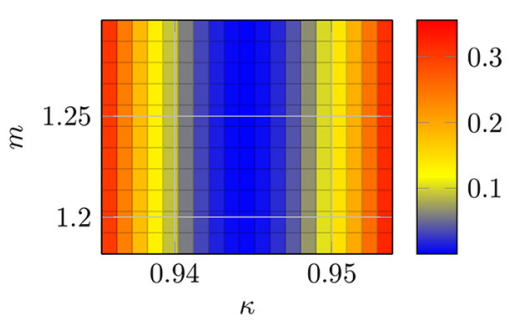

(b)

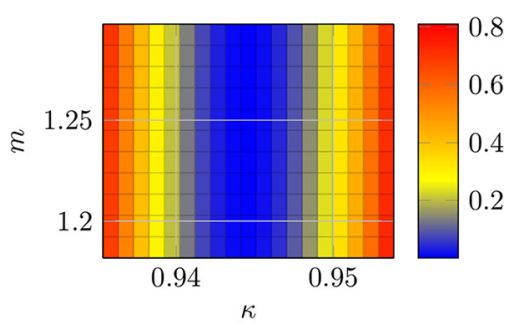

(c)

Figure 10. Data misfit values for a load point positioned at $2.1 \mathrm{~m}$ from the left anchorage having a magnitude of (a) I.0 kN, (b) $2.0 \mathrm{kN}$, and (c) $3.0 \mathrm{kN}$.

point magnitude and the displacement of the point of application of the load is almost linear and strain changes in the cable remain small, making the identification of the tension more difficult.

\section{Robustness with respect to sensor uncertainties (noise)}

The noise is usually tuned to be negligible with respect to the systematic bias. Moreover, the stochastic noise gets eliminated through time averaging over a typical interval of $10 \mathrm{~s}$, in order to get kinematic quantities that correspond to the static configuration. Hence, we do concentrate on the systematic bias and its propagation to the quantity of interest, that is, the cable tension.

To this end, the sensitivity of the tension to the sensor output can be computed by means of an optimality system. However, in this article, we will simply compute the error made in the tension assessment corresponding to an error of $\pm 10 \%$ made on the reference values of two displacement sensors placed at midspan and a third of the span. Numerical errors are introduced via two parameters $\alpha_{0.33}$ and $\alpha_{0.5}$ which multiply the simulated experimental data in $\mathfrak{J}^{\mathfrak{a}, \mathfrak{b}}(L, \kappa, m)$. Results of the error propagation through the inverse problem method are shown in Figure 11. Considering in a first time the line $\alpha_{0.33}=1.0$ corresponding to the case where only the displacement sensor located at midspan is affected by uncertainties of $\pm 10 \%$, we notice that the corresponding error in the tension assessment is of less than $4 \%$. Moreover, paying now attention to the blue points in the graph corresponding to an exact tension identification, we notice that errors made with opposite signs on the two sensors can reduce the overall error. Another comment concerns the lack of symmetry of the graph that is due to the way biases have been introduced: measured displacement is greater at a third of the span than at midspan when the point load is placed at a quarter of the span, and so are the biases introduced via the coefficient multiplication. In practice, an absolute bias per sensor would lead to an inverted symmetry since the relative error made on the greater measured displacement would be smaller than the one made on the smallest measured displacement. It confirms that it is better to place the displacement sensors near the point load, where the resulting displacements are larger. The robustness of the method can be assessed considering the extent of the area corresponding to an error in the tension assessment of less than $4 \%$ (in yellow and blue).

\section{Universal ambition of the method}

In order to show the universal applicability of the method, we choose to numerically assess the tension of cables spanning a large range of Irvine's parameters $\lambda$. Irvine's parameter definition can be found in Irvine, ${ }^{24}$ Chapter 2. As mentioned by its inventor, there is a cutoff value for $\lambda^{2}$ at 24 above which [the cable's] response has more in common with a heavy cable and below which it is akin to that expected of a taut string. The Irvine parameter is nondimensional and characterizes the static behavior of a cable. In theory, the method should perform as efficiently on cables with the same Irvine parameter.

Parametric studies were performed focusing on the length of a steel cable with a diameter of $50 \mathrm{~mm}$ hanging between two supports at the same level with a span $L_{s}=50 \mathrm{~m}$. The mass of $2 \mathrm{kN}$ at $s=L / 4$ and initial guesses $L=0.999 L_{s}, \kappa=1.02 \kappa_{t c}$, and $m=1.05 m_{t c}$ were the same for all the 201 tested cables. Corresponding safety factor values fall within the range 1.19-73. Two cases, namely, (1) and (2) are investigated: (1) solving the minimization problem considering all three parameters $\{L, \kappa, m\}$ as unknowns or (2) only the two parameters $\{L, \kappa\}$ are treated as unknowns. Differences between the two cases shed light on the role played by $m$ in the inverse problem. For both cases (1) and (2), the relative error made on $L$ is identical. Figure 12 shows the error made on the assessment of the cable 


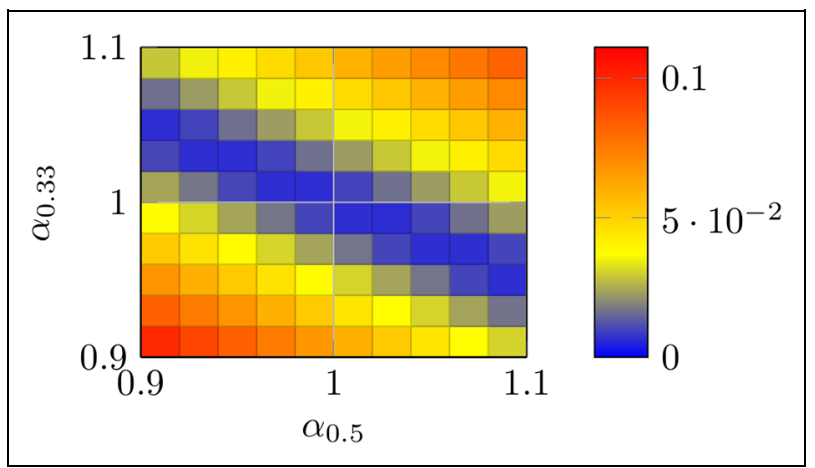

Figure I I. Error diffusion in tension assessment for parameters $\alpha_{0.33}$ and $\alpha_{0.5} \in[0.9, \mathrm{I} . \mathrm{I}]$ corresponding to uncertainties of $\pm 10 \%$ on the two displacement sensors measures at $s=7 \mathrm{~m}$ and $s=10.5 \mathrm{~m}$ for a point load of $1.0 \mathrm{kN}$ located at $s=5.4$.

parameters for a large variety of cables. Two general comments can be made. First, the proposed inverse method for tension assessment gives a relative error within $[-2.6: 1] \%$ for (1) and within $[-3.6: 1.4] \%$ for (2). Second, the first step of the procedure leads to a length assessment which is below $0.015 \%$ in all tested cases. The left part of the graph from $\log \lambda^{2}=-3$ to -0.6 mostly corresponds to taut cables with safety factors from 1.19 to 6 , respectively. As it can be seen, the tension estimation in that part of the graph is very accurate in both cases (1) and (2). Since the length and the tension are assessed correctly, the second step of the inverse problem has no impact on $\kappa$, as suggested by the taut cable example in section "Identification of the natural length of the cable." When $m$ is an unknown, that is, in case (1), the inverse problem leads to its accurate estimate, as predicted in Figure 8(a). The right part of Figure 12 shows that the tension assessment is improved in case (1), that is, when $m$ is a variable of the problem. Interestingly, this improvement is stronger for $\lambda^{2}$ values beyond the cutoff value equal to 24 .

Additional simulations were carried out following the same procedure but introducing a noise of $+1 \%$ and $-1 \%$ in all measured values. Figure 13 gives the corresponding relative errors made on the safety factor in both cases (1) and (2). For instance, the curve $\gamma(m,+1 \%)$ is a case (1) set of 201 simulations introducing a noise of $+1 \%$ in all measured parameters. As in the previous case, there is almost no difference in the tension assessment between cases (1) and (2) for taut cables while case (1) gives better results for sagged cables. It is worth noticing that the results shown in section "Robustness with respect to sensor uncertainties (noise)" can be extended to the whole range of taut cables and that the error diffusion is very limited.

At a computational level, the algorithm exhibits stable behavior and reaches convergence within 10-20 iterations for the length search and less than 10 iterations for the adjoint problem. With a standard laptop computer-i7-4510U CPU $2.00 \mathrm{GHz} / 2.6 \mathrm{GHz}, 8 \mathrm{Go}$ RAM - and using 2505 degrees of freedom, the overall

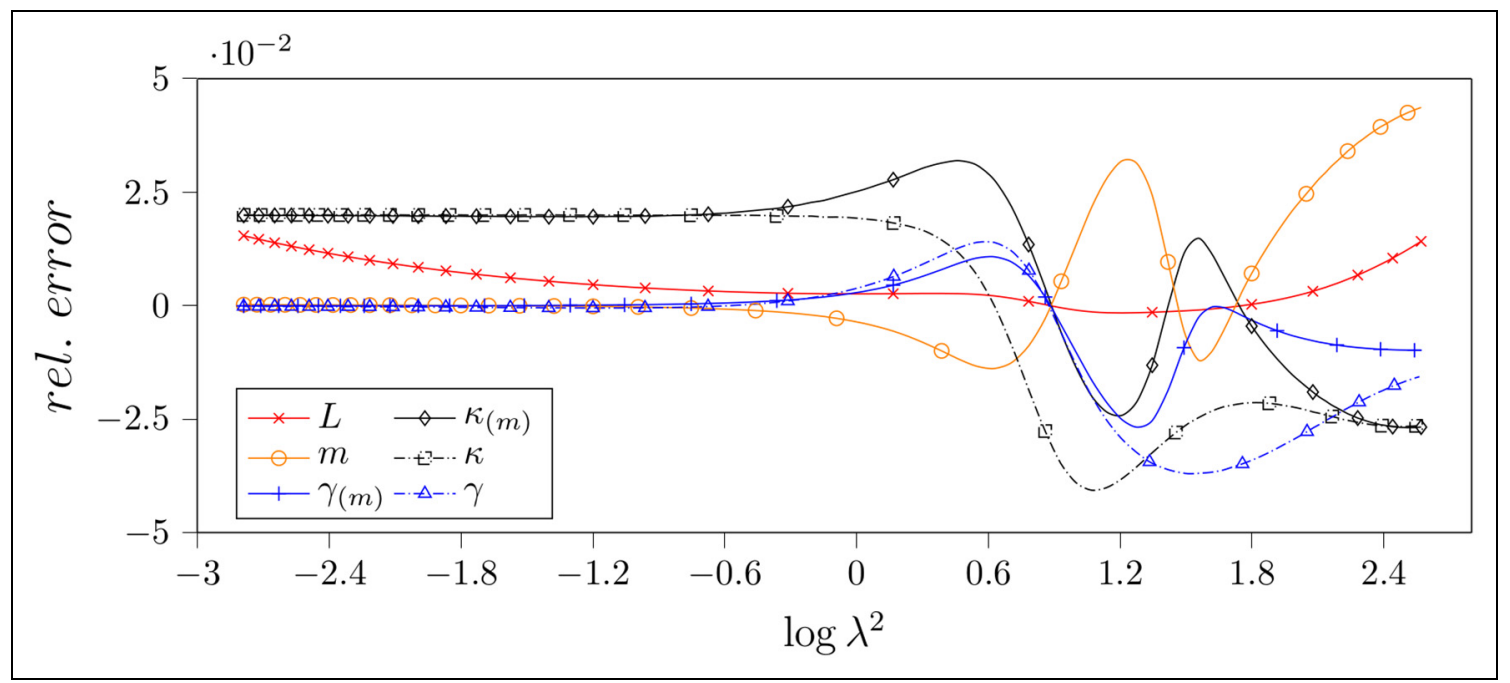

Figure 12. Relative error for several cable parameters w.r.t. $\log \lambda^{2}$. Parameters obtained considering $m$ as unknown of the problem have the notation $(m)$ next to them. Introduced errors on initial guesses $\kappa$ and $m$ are equal to $+2 \%$ and $+5 \%$, respectively. The initial guess used for the length is $L=0.999 L_{s}$ and its relative error was multiplied by 100 . For clarity purposes, only a few simulations are marked with a symbol.

Note: $\log 24=1.38$. 


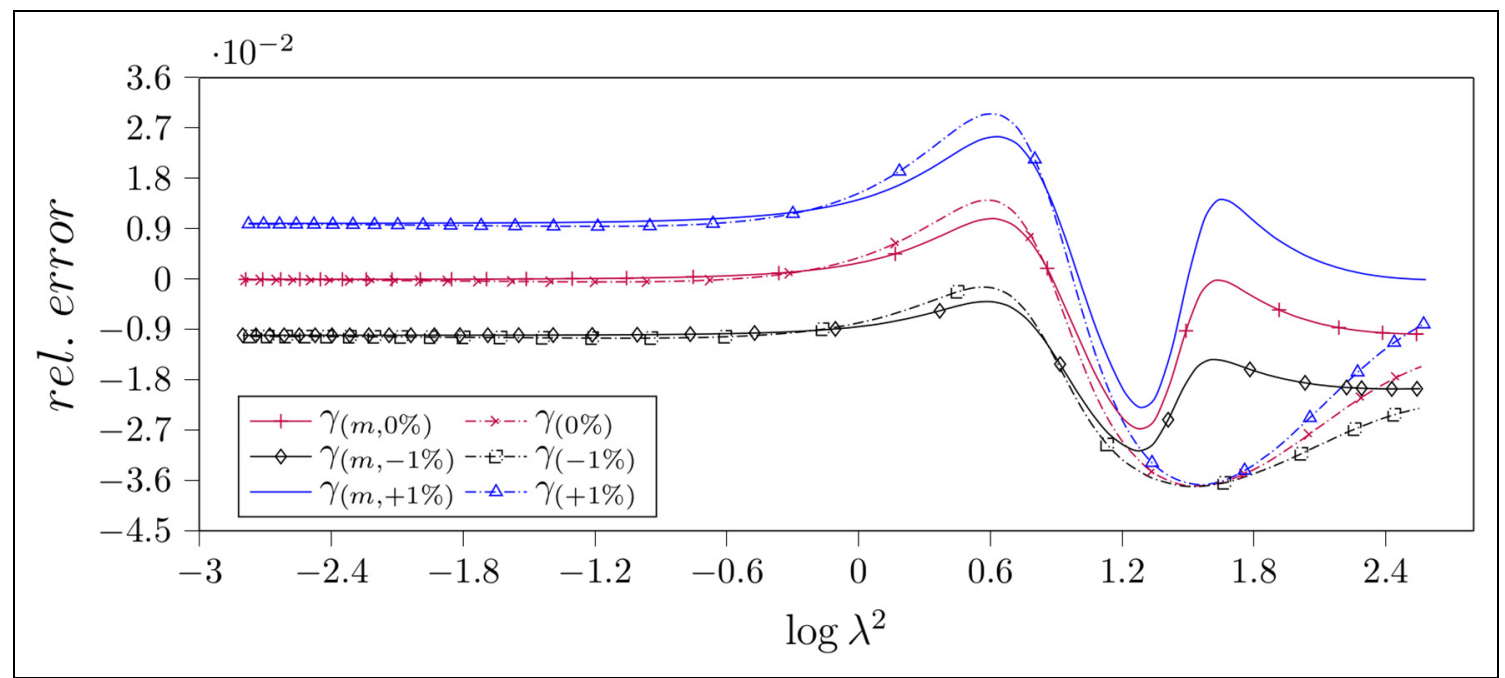

Figure 13. Relative error obtained for $\gamma$ w.r.t. $\log \lambda^{2}$. Information on the noise introduced in all measured parameters and on the dependency on $m$ is bracketed. Introduced errors on initial guesses $\kappa$ and $m$ are equal to $+2 \%$ and $+5 \%$, respectively. Initial guess used for the length is $L=0.999 L_{s}$. For clarity purposes, approximatively a tenth of the simulations are marked with a symbol. Note: $\log 24=1.38$.

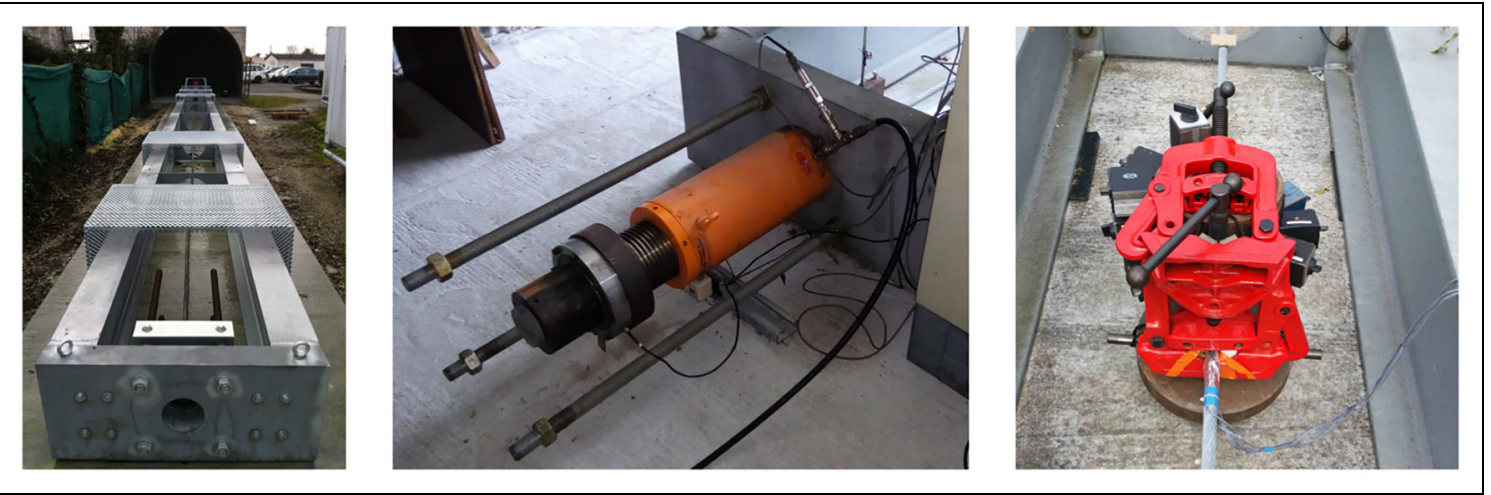

Figure 14. Test bench. From left to right: bench structure, jack and load cell, and additional mass to create a point load.

tension assessment does not take more than 3 min per cable.

\section{Experimental validation}

\section{Setup}

Experimental validation was conducted with a steel multilayered stranded cable $21 \mathrm{~m}$ long and $22 \mathrm{~mm}$ in diameter. The test protocol reproduces the two static configurations $a$ and $b$ of the cable for six different tension levels in configuration $a$. The point load is applied via a $86.120 \mathrm{~kg}$ mass of steel as depicted in Figure 14. In order to double-check the tension obtained via the load cell, dynamical testing was performed for each tension level. The monitoring system consists of the following items:

- Two accelerometers: DeltaTron type 4507 B 002 Brüel \& Kjær $\pm 70 \mathrm{~m} \mathrm{~s}^{-2}$ peak.

- Two displacement sensors: Intelligent-L300 Laser Sensor Keyence 160-450 mm.

- A load cell: FN2796 Measurement Specialties up to $400 \mathrm{kN}$.

- Four strain gauges from Tokyo Sokki Kenkyujo Co., Ltd.

Sensors are shown on Figures 14 and 15. A drawing of the setup appears on Figure 3 where the four strain sensors are placed at $18 \mathrm{~m}$ from the left anchorage. 


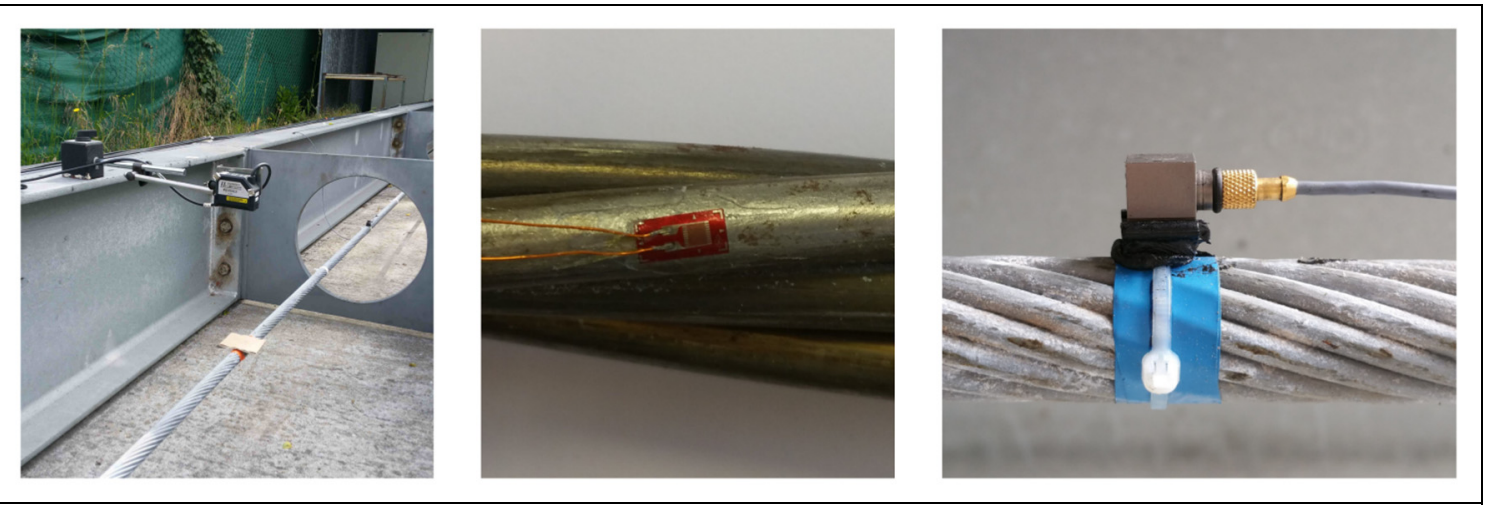

Figure I 5. Sensors used. From left to right: displacement laser sensor, strain gauge, and accelerometer.

Table I. Data obtained for six tension levels.

\begin{tabular}{|c|c|c|c|c|c|c|c|c|}
\hline Sensor & Parameters & Units & Tests & & & & & \\
\hline Load cell & Tension & $(\mathrm{kN})$ & 55 & 85 & 115 & 145 & 175 & 205 \\
\hline Distance meter & 1 & (m) & 21.733 & 21.747 & 21.763 & 21.777 & 21.794 & 21.809 \\
\hline 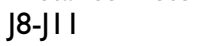 & $\Delta^{b}-\Delta^{a}$ & ( $\mu$ def) & 47.6 & 17.6 & $\mathrm{I} .4$ & 3 & 0.4 & 1.6 \\
\hline DI & $r_{1}^{1, b}-r_{1}^{1, a}$ & $(\mathrm{~mm})$ & -52.5 & -35.7 & -26.1 & -20.7 & -16.9 & -14.4 \\
\hline D2 & $r_{1}^{2, b}-r_{1}^{2, a}$ & $(\mathrm{~mm})$ & -64.2 & -43.3 & -31.7 & -24.8 & -20.3 & -17.5 \\
\hline
\end{tabular}

Table 2. Tension assessment results for the six tested tensions.

\begin{tabular}{lrrrrrr}
\hline & \multicolumn{1}{c}{2} & 2 & 3 & 4 & 5 & 6 \\
\hline$T_{\text {cell }}(\mathrm{kN})$ & 55.9 & 84.7 & 114.7 & 145.1 & 174.6 & 171.2 \\
$T_{\text {inv }}(\mathrm{kN})$ & 50.9 & 79.3 & 110.1 & -39.6 & -2.0 & 199.8 \\
$\mathrm{e}_{\text {inv }}(\%)$ & -8.9 & -6.4 & -4.0 & 135.7 & -2.9 \\
$T_{\text {dyn }}(\mathrm{kN})$ & 49.8 & 77.1 & 107.1 & -6.8 & -5.6 & 193.3 \\
$\mathrm{e}_{\text {dyn }}(\%)$ & -10.9 & -9.0 & -6.7 & & -6.0 \\
\hline
\end{tabular}

The following protocol has been applied at six different tension levels from 55 to $205 \mathrm{kN}$ :

1. Cable installation up to a specific tension (load cell).

2. Static measurements.

3. Mass positioning.

4. Static measurements.

5. Mass removal.

Contemporaneously to the experimental validation of our method, dynamical testing based on taut string theory was performed for double-checking purposes: FFTs were applied on the acceloremetric data in order to obtain the cable vertical natural frequencies. In the taut string theory, the explicit relationship between the (constant) tension $T$ in the cable and its first natural frequency $f_{1}$ is simply $T=4 f_{1}^{2} l^{2} \rho A$, where $l$ is the length of the cable which is also its span.
The use of the string theory is justified for validation purposes since it can be shown that in our testing conditions, its accuracy is almost constant through all the tests and is driven by the precision of the knowledge of $\rho A$. In fact, even for the lowest tested tension, we have: $d T / T=2 d l / l+2 d f_{1} / f_{1}+d \rho A / \rho A \approx d \rho A / \rho A$. For the test, we assume that the mass per unit length of cable is equal to $\rho A=7810 \times 0.75 \pi \times 0.011^{2} \mathrm{~kg} \mathrm{~m}^{-1}$, in accordance with the commonly used fill ratio for stranded cables.

\section{Results}

Data from the tests are reported in Table 1. Post-processing data using both the string theory and our inverse problem method lead to the results shown in Table 2. Paying attention to the results obtained with the dynamical testing methods, we notice a large variation of the accuracy of the method over the tests from 
Table 3. Tension assessment results for the six tested tensions.

\begin{tabular}{|c|c|c|c|c|c|c|}
\hline & I & 2 & 3 & 4 & 5 & 6 \\
\hline$T_{\text {ref }}(\mathrm{kN})$ & 53.0 & 82.0 & 113.9 & 143.9 & 175.3 & 205.7 \\
\hline$T_{i n v}(\mathrm{kN})$ & 50.9 & 79.3 & I I0.I & 139.6 & 171.2 & 199.8 \\
\hline$e_{i n v}(\%)$ & -3.9 & -3.3 & -3.3 & -2.9 & -2.4 & -2.9 \\
\hline$T_{\text {dyn }}(\mathrm{kN})$ & 49.8 & 77.1 & 107.I & 135.2 & 164.8 & 193.3 \\
\hline $\mathrm{e}_{\text {dyn }}(\%)$ & -6.0 & -6.0 & -6.0 & -6.0 & -6.0 & -6.0 \\
\hline
\end{tabular}

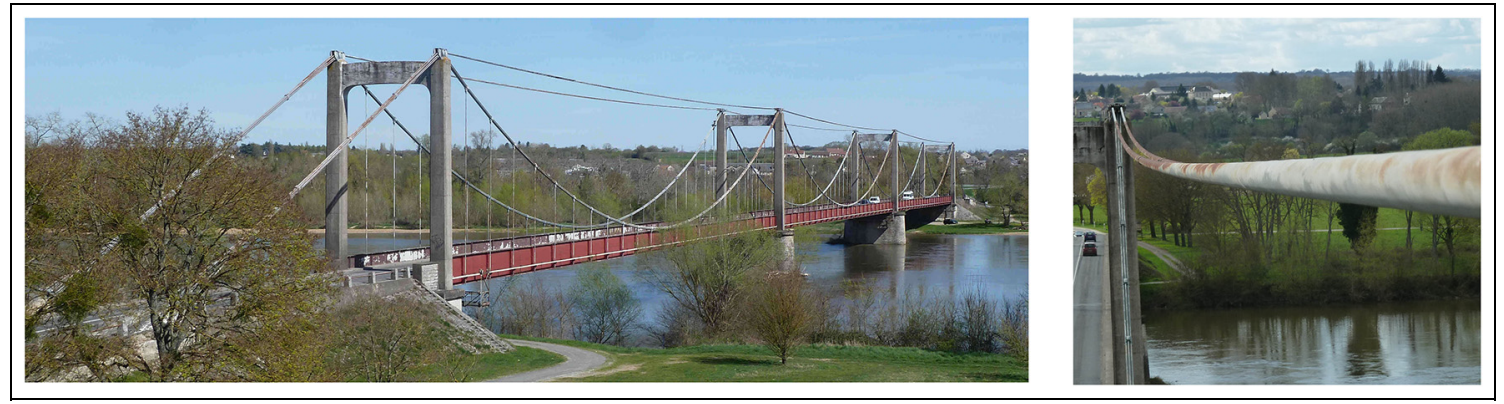

Figure 16. Bonny-sur-Loire (Loiret, France) suspension bridge with top cables.

$-10.9 \%$ to $-6.0 \%$. This result is in contradiction with the expected constant accuracy of the method, as previously discussed. According to the calibration tests, the accuracy of the load cell is close to $1 \mathrm{kN}$ for high values of tensions and about $3 \mathrm{kN}$ for low tensions. Trusting the highest load measurement at $205 \mathrm{kN}$, it is possible to calibrate the dynamical method by correcting the mass per unit length of cable to obtain a reference tension $T_{r e f}$ in the cable for all tests. We report in Table 3 the corresponding values.

At it can be seen, the correction leads now to a consistent and constant error for the dynamical tension assessment. Using the load cell and the test at the highest level of tension, we are able to say that our estimation of the mass per unit length of cable was a $6 \%$ underestimation. More importantly, the proposed inverse problem achieves good accuracy in finding the cable tension for all the six tension levels tested despite the poor knowledge we had on the mass per unit length of cable.

As mentioned in section "Universal ambition of the method," the ambition of the proposed method is to be able to assess the tension of all different types of cables (cables with taut profile, sagged profile, tensed or not). Focusing on the tension assessment of taut cables, very light frequency-based methods using contactless sensors exist ${ }^{4}$ but require a refined knowledge of the mass per unit length of cable. In turn, these frequency-based methods can serve to identify the mass per unit length of cable provided that the tension is known. The proposed inverse method can therefore be used to calibrate these methods and enhance their precision.

\section{Application to three top cables of a suspension bridge}

\section{Top cables}

The method was used on three top cables of a 77-yearold French suspension bridge crossing Loire river at Bonny-sur-Loire (see Figure 16). According to the project design from 1948 found in the bridge owner's archives, top cables are $120 \mathrm{~m}$ long and $80 \mathrm{~mm}$ in diameter (cross-sectional area $3765 \mathrm{~mm}^{2}$ ). They are tensed at $1071 \mathrm{kN}$ and their mass per unit length of cable is $31.719 \mathrm{~kg} \mathrm{~m}^{-1}$. Before applying our method, three comments are in order: (1) the data found in the archives are not always available and it is very difficult to know if the construction products delivered on the job site 77 years ago are the one described in the project design, (2) measured diameters are around $82 \mathrm{~mm}$ and would also match commonly used cables with a diameter of $81.6 \mathrm{~mm}$, a cross-sectional area of $3926 \mathrm{~mm}^{2}$, and a mass per unit length of cable equals to $33.085 \mathrm{~kg}$ $\mathrm{m}^{-1}$, and (3) the corrosion protection complex (pitchbased mixture inside the cable and paint) is no longer efficient and due to the age of the cables, their mass per unit length is deemed overestimated by both previously mentioned values. 
Table 4. Test results and tension assessment via static inverse method.

\begin{tabular}{|c|c|c|c|c|c|c|c|c|c|}
\hline \multirow{2}{*}{$\frac{\text { Cable name }}{\text { Load }(\mathrm{N})}$} & \multicolumn{3}{|c|}{ AmTICTI } & \multicolumn{3}{|c|}{ AmTICT2 } & \multicolumn{3}{|c|}{ AvTICTI } \\
\hline & 1005 & 1573 & 1883 & 1135 & 1616 & 2062 & 1063 & |57| & 2074 \\
\hline Displacement $(\mathrm{mm})$ & -38.5 & -61.0 & -72.6 & -42.7 & -61.2 & -77.5 & -41.6 & -60.1 & -79.5 \\
\hline$T_{\text {inv }}(\mathrm{kN})$ & 669.8 & 650.6 & 653.7 & 688.3 & 676.3 & 680.7 & 646.0 & 666.5 & 658.2 \\
\hline
\end{tabular}

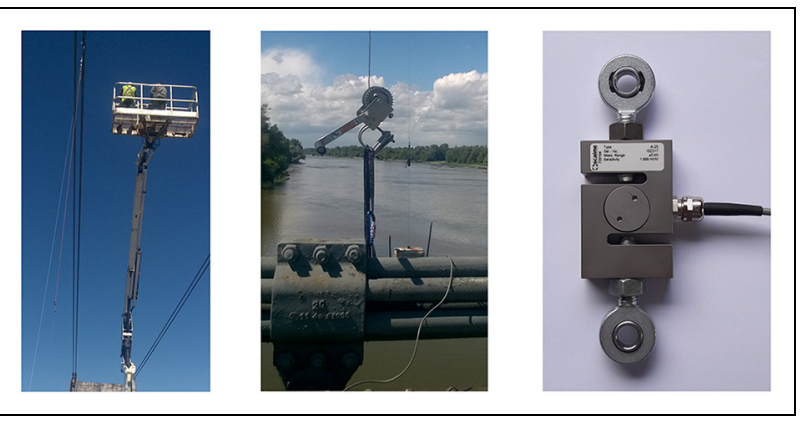

Figure 17. Application of the inverse method on a real suspension bridge.

Because for taut cables the displacement part $J_{\text {disp }}$ of the functional $\mathfrak{J}^{\mathfrak{a}, \mathfrak{b}}$ is preponderant, a variant of the method was formulated so as to comply with the following light and cheap monitoring system consisting in a TME P-15A displacement sensor placed at midspan plus a force sensor SCAIME-K25 $0-5 \mathrm{kN}$. The load was applied at midspan via a manual winch shown in Figure 17. Three load steps were applied on each cable at 1.0, 1.5, and $2 \mathrm{kN}$. Table 4 gives the corresponding obtained displacements and calculated tensions.

For comparison purposes, first frequencies of vibration were measured and two traditional frequencybased methods were applied to assess the tension in the cables: namely, the string theory method ${ }^{1}$ and the method proposed by Zui et al. ${ }^{2}$ Table 6 summarizes the main physical characteristics of the tested cables and can be found in Appendix 1. Found first frequencies are almost the same for the three cables and we therefore expect to find similar tensions via the frequencybased methods. The first frequencies $f_{1}$ are quite small and at the lower limit of the frequency range of the accelerometer used to perform the tests. Differences between higher modes $f_{3}-f_{2}$ and $f_{4}-f_{3}$ suggest that first frequencies $f_{1}$ are overestimated. As a result, the two frequency-based methods were also applied on higher frequency orders. Table 5 presents the obtained tensions when the first four modes are used. It can be clearly seen that the first frequencies lead to an overestimation of the tension with both dynamical methods. Discarding tensions calculated with mode 1 , we notice that the tensions assessed with the LPC 35 are close to
$700 \mathrm{kN}$, the ones given by Zui et al.'s ${ }^{2}$ method are around $680 \mathrm{kN}$ while the static inverse method gives tensions around $660 \mathrm{kN}$. The mass per unit length of cable that has been used comes from the design project and is a standard value accounting for the overall weight of the cable (steel plus a standard corrosion protection weight commonly used when the bridge was built). Taking into account aging factors, it is reasonable to consider that actual masses per unit length of cables are smaller, especially because visual inspection showed that the internal corrosion protection product used is leaking out of a large number of cables. The corresponding variation in the mass per unit length of cable is around $5 \%$. Therefore, under this hypothesis, LCPC35 and Zui et al. ${ }^{2}$ would yield around 665 and $646 \mathrm{kN}$, respectively. Hence, assuming the validity of this hypothesis, the static inverse method would lead to more accurate results than the frequency-based method. In this specific case, it would have been interesting to truly assess the mass per unit length of cable by applying the inverse method in its full version, that is, as it is described in section "Inverse problem."

\section{Affordability}

It might look very expensive to advocate the use of added masses and displacement sensors that need to be attached to bridge deck and the cable. However, current maintenance procedures impose periodic man power over the bridge with often heavy equipment (visual inspection). The tension evaluation process that we propose here only needs to be carried out every time a large maintenance or repair operation is done on the bridge such as cable painting renewal. It can thus be considered as lean process that only incurs marginal extra cost. The outcome of the method might be viewed as the necessary pre-processing stage of the standard and very cheap standard dynamical testing methods as described in Gentile. ${ }^{4}$

\section{Conclusion}

In this article, we proposed an innovative tension identification procedure that relies on limited prior knowledge of the cable, use of current practice instrumentation schemes, and an inverse static problem 
Table 5. Comparison between frequency-based and inverse static tension assessment methods.

\begin{tabular}{|c|c|c|c|c|c|c|c|c|c|}
\hline \multirow{2}{*}{$\begin{array}{l}\text { Method } \\
\text { Mode number }\end{array}$} & \multicolumn{4}{|c|}{ LPC35 } & \multicolumn{4}{|c|}{ Zui et al. ${ }^{2}$} & \multirow{2}{*}{$\begin{array}{l}\text { Static inverse method } \\
\text { Mean } T_{i n v}\end{array}$} \\
\hline & I & 2 & 3 & 4 & I & 2 & 3 & 4 & \\
\hline AmTICTI (kN) & 845 & 691 & 695 & 697 & 782 & 678 & 720 & 684 & 658 \\
\hline AmTICT2 (kN) & 845 & 702 & 702 & 708 & 730 & 678 & 682 & 684 & 682 \\
\hline AvTICTI (kN) & 796 & 691 & 733 & 697 & 730 & 690 & 690 & 695 & 657 \\
\hline
\end{tabular}

based on a nonlinear mixed formulation of elastic cable equilibrium. More precisely, several novelties in the presented tension identification should be here highlighted. To the authors' knowledge, tension identification in cables is seldom addressed via an inverse static approach. Moreover, only a few works consider the length, the stiffness, and the mass per unit length of cable as unknowns of the problem despite their importance and the fact that they are only roughly known in practice. From a modeling point of view, the use of a mixed formulation for a geometrically exact cable is also unusual in the literature regardless of the advantages one could reap from the direct calculation of strains and stresses viewed as primary variables. ${ }^{15}$ The inverse problem for tension identification is obtained via a data misfit functional $\mathfrak{J}^{\mathfrak{a}, \mathfrak{b}}(L, \kappa, m)$ relying on the differences in terms of displacements and strains measured at a few points on the cable between two static configurations, thus alleviating to a large extent the systematic bias of standard sensors as well as systematic modeling errors due to the lack of accurate knowledge of some physical parameters. Pure noise is also cured from data by time-averaging or low-pass filtering since static quantities are considered. Parametric studies of $\mathfrak{J}^{\mathfrak{a}, \mathfrak{b}}$ have been presented for a simulated 21-m long, flat cable with a diameter of $22 \mathrm{~mm}$. Despite the ill-posedness of the inverse problem caused by the multiplicity of its solutions, it has been shown that the correct tension could be found following a two-step procedure. First, fixing the axial stiffness and the mass per unit length at their initial guess values, the length $L$ of the cable is approximately found via a simple line search algorithm using finite differences to estimate the functional derivative of $\mathfrak{J}^{\mathfrak{a}, \mathfrak{b}}$ with respect to $L$. Second, the other two physical parameters are assessed using an adjoint method for which the direct problem, the adjoint problem, and parameter sensitivities are found as derivatives of a Lagrangian functional with respect to dual variables, primary variables, and parameters, respectively. Numerical simulations given at the end of section "Performance assessment of the inverse methodology" illustrate the potential of the method: even if physical parameters of the cable are not correctly assessed, their evaluation discrepancies are mutually canceled in the minimization problem leading to the correct tension identification. In theory and for taut cables, the mass per unit length of cable could also be correctly assessed. An experimental validation has been carried out with a 21-m long, multilayered stranded cable having a diameter of $22 \mathrm{~mm}$ undergoing six different tension levels from 55 up to $205 \mathrm{kN}$. A comparison with a traditional dynamical testing showed the reliability of the proposed method and its interest; tensions were correctly assessed within less than $4 \%$ error without requiring a fine knowledge of the cable's stiffness and mass per unit length. Last but not least, the method has been successfully tested on three $120-\mathrm{m}$ long top cables of a real bridge and showed its robustness for on-site applications.

\section{Acknowledgements}

The authors acknowledge $\mathrm{Mr}$ Christian Lardeux (Département du Loiret) for giving full access to the Bonnysur-Loire bridge and its archives, and Mr John Dumoulin and Mr Rémi Piednoir (Cerema Sud-Ouest) for the role they played in the experimental validation of the method.

\section{Declaration of conflicting interests}

The author(s) declared no potential conflicts of interest with respect to the research, authorship, and/or publication of this article.

\section{Funding}

The author(s) received no financial support for the research, authorship, and/or publication of this article.

\section{ORCID iD}

Arnaud Pacitti (DD https://orcid.org/0000-0003-1247-9832

\section{References}

1. Robert JL. LPC 35: Mesure de la tension des câbles par vibration: Techniques et Méthodes des Laboratoires des Ponts et Chaussées [LPC 35: dynamic cables' tension measurement]. Technical report, 1993, https://www.ifstt ar.fr/fileadmin/user_upload/editions/lcpc/MethodeDEssai/ MethodeDEssai-LCPC-ME35.pdf

2. Zui H, Shinke T and Namita Y. Practical formulas for estimation of cable tension by vibration method. $J$ Struct Eng: ASCE 1996; 122(6): 651-656. 
3. Bouton E and Crémona C. Note sur la méthode d'essai LCPC [note on the LCPC testing method]. Technical report, LCPC, Paris, 2003.

4. Gentile C. Deflection measurement on vibrating stay cables by non-contact microwave interferometer. $N D T \& E$ Int 2010; 43: 231-240.

5. Kangas S, Helmicki A, Hunt V, et al. Identification of cable forces on cable-stayed bridges: a novel application of the MUSIC algorithm. Exp Mech 2010; 50(7): 957-968.

6. Lardiès $\mathrm{J}$ and Ta MN. Modal parameter identification of stay cables from output-only measurements. Mech Syst Signal Pr 2011; 25: 133-150.

7. Caetano E. On the identification of cable force from vibration measurements. In: Proceedings of the IABSEIASS symposium, London, 20-23 September 2011.

8. Li S, Reynders E, Maes K, et al. Vibration-based estimation of axial force for a beam member with uncertain boundary conditions. J Sound Vib 2013; 332: 795-806.

9. Siegert D, Dieng L, Brevet P, et al. Parameter identification of a hanger or stay cable model based on measured resonant frequencies. In: Proceedings of the international conference on experimental vibration analysis for civil engineering structures (EVACES'05), Bordeaux, 26-28 October 2005 .

10. Park S, Choi S, Oh ST, et al. Identification of the tensile force in high-tension bars using modal sensitivities. Int $J$ Solids Struct 2006; 43(10): 3185-3196.

11. Kim BH and Park T. Estimation of cable tension force using the frequency-based system identification method. J Sound Vib 2007; 304: 660-676.

12. Bellino A, Marchesiello S, Fasana A, et al. Cable tension estimation by means of vibration response and moving mass technique. Méc Ind 2010; 11(6): 505-512.

13. Lepidi M, Gattulli V and Vestroni F. Damage identification in elastic suspended cables through frequency measurement. J Vib Control 2009; 15(6): 867-896.

14. Joaquim AR, Cismasiu C, Santos FA, et al. Estimation of the tensile force in the stay-cables of Salgueiro Maia bridge using ambient vibration tests. In: Proceedings of the international symposium on the dynamics and aerodynamics of cables (ISDAC'2017), Faculty of Engineering of the University of Porto (FEUP), Porto, 30-31 October 2017. Porto: FEUP.

15. Pacitti A. Nonlinear modeling of elastic cables: experimental data-based tension identification via static inverse problem. PhD Thesis, University of Paris-Est, Marnela-Vallée; Sapienza University of Rome, Rome, 2016.

16. Logg A, Mardal KA, Wells GN, et al. Automated solution of differential equations by the finite element method. Berlin; Heidelberg: Springer, 2012.

17. Hadamard J. Sur les problèmes aux dérivées partielles et leur signification physique. Princeton Univ Bullet 1902; 13: 49-52.

18. Bui HD. Inverse problems in the mechanics of materials: an introduction. Paris: Éditions Eyrolles, 1994.

19. Kern M. Problèmes inverses: aspects numériques (Lecture), 2002, https://hal.archives-ouvertes.fr/cel-00168393

20. Antman SS. Nonlinear problems of elasticity. 2 nd ed. New York: Springer, 2005.
21. Lacarbonara W. Nonlinear structural mechanics: theory, dynamical phenomena, and modeling. New York: Springer, 2013.

22. He J. Equivalent theorem of Hellinger-Reissner and $\mathrm{Hu}-$ Washizu variational principles. J Shanghai Univ: Engl Ed 1997; 1(1): 36-41.

23. Barzilai $\mathbf{J}$ and Borwein $\mathbf{J M}$. Two-point step size gradient methods. IMA J Numer Anal 1988; 8(1): 141-148.

24. Irvine HM. Cable structures. Mineola, NY: Dover Publications, 1984.

\section{Appendix I}

\section{Newton-Raphson iterative procedure}

In practice, we solve the nonlinear problem via an iterative procedure. At each step $k$, the linearized problem to be solved is

Find $\mathfrak{d} \breve{r} \in \mathbb{V}_{0}, \mathfrak{d} \breve{\Delta} \in \mathbb{P}_{\Delta}$ and $\mathfrak{d} \breve{T} \in \mathbb{P}_{\breve{T}}$ such that

$$
\begin{aligned}
& \forall \delta \breve{\boldsymbol{r}} \in \mathbb{V}_{0}, \delta \breve{\Delta} \in \mathbb{P}_{\Delta} \text { and } \delta \breve{T} \in \mathbb{P}_{\breve{T}} \\
& \mathfrak{d} \delta J\left(\breve{\boldsymbol{r}}_{k}, \breve{\Delta}_{k}, \breve{T}_{k}\right)=-\delta J\left(\breve{\boldsymbol{r}}_{k}, \breve{\Delta}_{k}, \breve{T}_{k}\right)
\end{aligned}
$$

with the update formulas

$$
\begin{aligned}
& \breve{\Delta}_{k+1}=\breve{\Delta}_{k}+\mathfrak{d} \breve{\Delta} \\
& \breve{\boldsymbol{r}}_{k+1}=\breve{\boldsymbol{r}}_{k}+\mathfrak{d} \breve{\boldsymbol{r}} \\
& \breve{T}_{k+1}=\breve{T}_{k}+\mathfrak{d} \breve{T}
\end{aligned}
$$

The second variation $\mathfrak{d} \delta J(\breve{\boldsymbol{r}}, \breve{\Delta}, \breve{T})$ is given by

$$
\begin{array}{r}
\mathfrak{d} \delta J=\int_{0}^{L}\left[(E A \mathfrak{d} \breve{\Delta}-\mathfrak{d} \breve{T}) \delta \breve{\Delta}+\mathfrak{d} \breve{T} \breve{\boldsymbol{r}}_{s} \cdot \delta \breve{\boldsymbol{r}}_{s}\right. \\
\left.-\left(\mathfrak{d} \breve{\Delta}-\breve{\boldsymbol{r}}_{s} \cdot \mathfrak{d} \breve{\boldsymbol{r}}_{s}\right) \delta \breve{T}+\breve{T} \mathfrak{d} \breve{\boldsymbol{r}}_{s} \cdot \delta \breve{\boldsymbol{r}}_{s}\right] d s
\end{array}
$$

and corresponds to the linearization of the nonlinear problem (9) around $\{\breve{\boldsymbol{r}}, \breve{\Delta}, \breve{T}\}$.

Integration by parts of equation (22)

$$
\begin{aligned}
& \int_{0}^{L}\left[\left(\kappa \breve{\Delta}^{b}-\breve{T}^{b}\right) \delta \breve{\Delta}^{* b}-\left(\left(\breve{T}^{b} \breve{\boldsymbol{r}}_{s}^{b}\right)_{s}+\boldsymbol{f}^{b}\right) \cdot \delta \breve{\boldsymbol{r}}^{* b}\right. \\
& \left.-\left(\breve{\Delta}^{b}-\breve{\Delta}^{e, b}\right) \delta \breve{T}^{* b}\right] d s+\left[\breve{T}^{b} \breve{\boldsymbol{r}}_{s}^{b} \cdot \delta \widetilde{\boldsymbol{r}}^{* b}\right]_{0}^{L}=0
\end{aligned}
$$

where the following boundary conditions have to be applied: $\delta \widetilde{\boldsymbol{r}}^{* b}(0)=\delta \boldsymbol{r}^{* b}(L)=o$. Same equations with $a$ superscripts hold true.

Integration by parts of equation (24) 


$$
\begin{gathered}
\int_{0}^{L}\left(\left(\breve{T}^{b} \breve{\boldsymbol{r}}_{s}^{* b}+\breve{T}^{* b} \breve{\boldsymbol{r}}_{s}^{b}\right)_{s}+\boldsymbol{f}\right) \cdot \delta \breve{\boldsymbol{r}}^{b} d s \\
=\int_{0}^{L}\left[\left(\kappa \breve{\Delta}^{* b}-\breve{T}^{* b}-f^{\Delta}\right) \delta \breve{\Delta}^{b}\right. \\
\left.+\left(\breve{\boldsymbol{r}}_{s}^{b} \cdot \breve{\boldsymbol{r}}_{s}^{* b}-\breve{\Delta}^{* b}\right) \delta \breve{T}^{b}\right] d s
\end{gathered}
$$

where the following boundary conditions were used: $\delta \boldsymbol{r}^{b}(0)=\delta \boldsymbol{r}^{b}(L)=\boldsymbol{o}$. Same equations with $a$ superscripts hold true.

\section{Backtracking the length of the cable}

The pseudocode to backtrack the length of the cable via finite differentiation of the data misfit $\mathfrak{J}^{\mathfrak{a}, \mathfrak{b}}(L)$ is given in Algorithm 1.

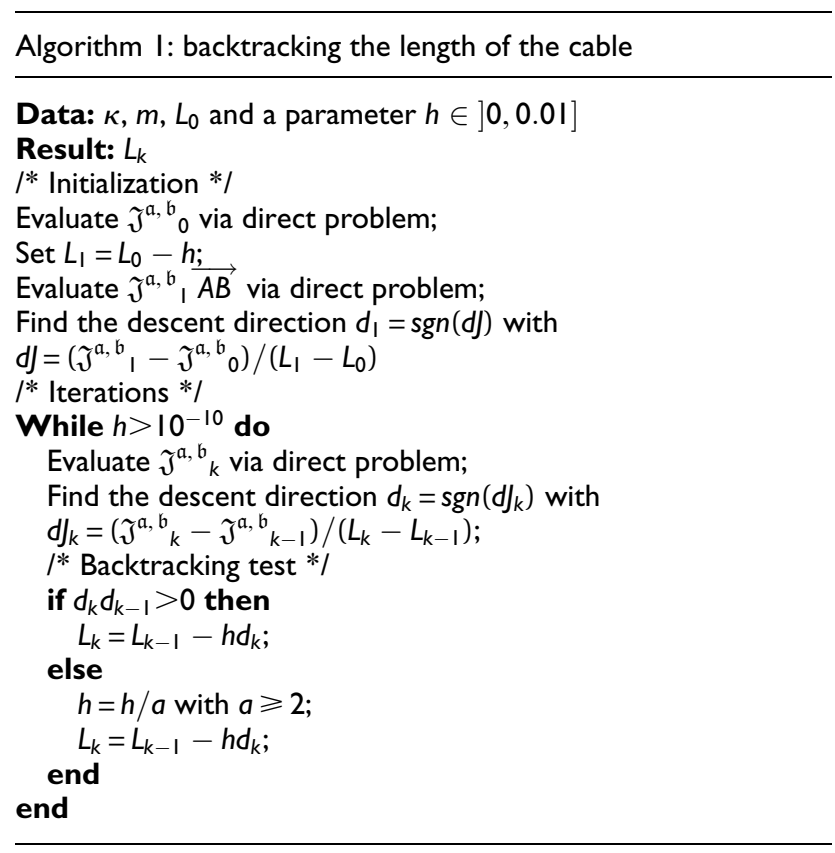

\section{Barzilai-Borwein algorithm}

Using the notations, $\boldsymbol{x}={ }^{t}[\kappa, m]$ and $\boldsymbol{g}=\nabla_{x} \mathfrak{L}$. The Barzilai-Borwein algorithm pseudocode can be found in Algorithm 2.
Algorithm 2: Barzilai-Borwein algorithm used for the minimization problem

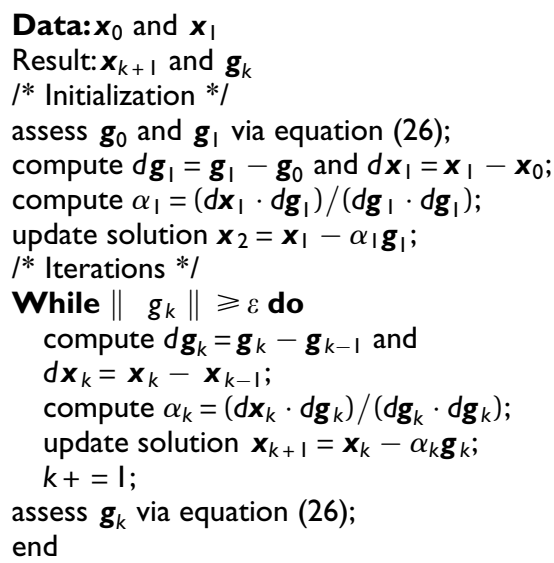

\section{Data used for frequency-based tension assessment methods}

Physical characteristics of the tested cables are shown in Table 6.

Table 6. Main physical characteristics of the tested cables.

\begin{tabular}{llll}
\hline Cable name & AmTICTI & AmTICT2 & AvTICTI \\
\hline$\rho A\left(\mathrm{~kg} \mathrm{~m}^{-1}\right)$ & 31.719 & 31.719 & 31.719 \\
$L(\mathrm{~m})$ & 120 & 120 & 120 \\
$E(\mathrm{GPa})$ & 150 & 150 & 150 \\
$f_{1}(\mathrm{~Hz})$ & 0.68 & 0.68 & 0.66 \\
$f_{2}(\mathrm{~Hz})$ & 1.23 & 1.24 & 1.23 \\
$f_{3}(\mathrm{~Hz})$ & 1.85 & 1.86 & 1.9 \\
$f_{4}(\mathrm{~Hz})$ & 2.47 & 2.49 & 2.47 \\
\hline
\end{tabular}

